\title{
COMMUTATORS AND COMMUTATOR SUBGROUPS OF FINITE $p$-GROUPS
}

\author{
RAHUL KAUSHIK AND MANOJ K. YADAV
}

\begin{abstract}
We present a classification of finite $p$-groups $G$ with $\gamma_{2}(G)$, the commutator subgroup of $G$, of order $p^{4}$ and exponent $p$ such that not all elements of $\gamma_{2}(G)$ are commutators.
\end{abstract}

\section{INTRODUCTION}

This paper is devoted to the study of finite $p$-groups $G$ with $\gamma_{2}(G)$ of order $p^{4}$ and exponent $p$ such that $\mathrm{K}(G)=\gamma_{2}(G)$, where $\gamma_{2}(G)$ denotes the commutator subgroup of $G$ and $\mathrm{K}(G):=$ $\{[x, y] \mid x, y \in G\}$. A similar study for finite $p$-groups $G$ with $\gamma_{2}(G)$ minimally generated by at most 3 elements has already been carried out in the past. Rodney 20] proved that $\mathrm{K}(G)=\gamma_{2}(G)$ for a nilpotent group $G$ with cyclic commutator subgroup. He 21] also proved that $\mathrm{K}(G)=\gamma_{2}(G)$ for all finite groups $G$ with $\gamma_{2}(G)$ elementary abelian of order $p^{3}$ for any prime integer $p$. Guralnick [7] considered finite groups $G$ such that $\gamma_{2}(G)$ is an abelian $p$ group minimally generated by at most 3 elements, and proved that $\mathrm{K}(G)=\gamma_{2}(G)$ for such groups, where $p \geq 5$. In a series of two papers Fernandez-Alcober and Heras [5], and Heras [12], respectively, it is proved that $\mathrm{K}(G)=\gamma_{2}(G)$ for a finite $p$-group $G$ such that $\gamma_{2}(G)$ is generated by 2 elements, and for a finite $p$-group $G, p \geq 5$, such that $\gamma_{2}(G)$ is generated by 3 elements. A generalization of some of these results for commutators of higher weights has also been studied in the literature. The interested reader may refer to [2, 8, 9, 13].

Examples of groups $G$ of order $p^{8}$ and nilpotency class 2 such that $\mathrm{K}(G) \neq \gamma_{2}(G)$ were constructed by Macdonald [17, Exercise 5, Page 78]. Such examples of groups of order $p^{6}, p \geq 5$, having nilpotency class 4 and groups of order $2^{7}$ having nilpotency class 3 were constructed by Kappe and Morse in [15], where the authors proved that $\mathrm{K}(G)=\gamma_{2}(G)$ for all $p$-groups of order at most $p^{5}$ and for all 2 -groups of order at most $2^{6}$. Some more details on the topic can be found in [16]. So it is evident that a condition only on the prime $p$ for a finite $p$-group $G$ can not ensure that $\mathrm{K}(G)=\gamma_{2}(G)$. The counter examples above of order $2^{8}$ and $p^{6}, p \geq 5$, admit elementary abelian commutator subgroups of order $2^{4}$ and $p^{4}$ respectively. This motivates us to look at the classification of finite $p$-groups $G$ with $\gamma_{2}(G)$ elementary abelian of rank 4 such that $\mathrm{K}(G) \neq \gamma_{2}(G)$. This study will also reveal patterns for future investigation on the topic. It follows from Lemma 2.2 that the property under consideration is invariant under isoclinism of groups (see Section 2 for the definition), which is an equivalence relation. As observed in the next section, each isoclinism family admits a group $G$ such that $\mathrm{Z}(G) \leq \gamma_{2}(G)$, where $\mathrm{Z}(G)$ denotes the center of $G$. In the following result we provide the desired characterization of finite $p$-groups (upto isoclinism) whose commutator subgroups are of order $p^{4}$ and exponent $p \geq 3$.

2010 Mathematics Subject Classification. Primary 20D15, $20 \mathrm{~F} 12$.

Key words and phrases. commutator, commutator subgroup, $p$-group. 
Theorem A. Let $G$ be a finite p-group with $\mathrm{Z}(G) \leq \gamma_{2}(G)$ and $\gamma_{2}(G)$ of order $p^{4}$ and exponent $p \geq 3$. Then $\mathrm{K}(G) \neq \gamma_{2}(G)$ if and only if one of the following holds:

(1) $G$ is of order $p^{6}$ and nilpotency class 4 with $|\mathrm{Z}(G)|=p^{2}$.

(2) $G$ is of order $p^{7}$ and nilpotency class 3 with $|\mathrm{Z}(G)|=p^{3}$.

(3) $G$ is of order $p^{8}$ and nilpotency class 2 along with one of the following:

(3a) $G$ admits a non-central element whose centralizer in $G$ is a maximal subgroup.

(3b) $G$ is of conjugate type $\left\{1, p^{2}, p^{3}\right\}$ and admits no generating set $\left\{x_{1}, x_{2}, x_{3}, x_{4}\right\}$ such that $\left[x_{1}, x_{2}\right]=1=\left[x_{3}, x_{4}\right]$.

Moreover, if $\mathrm{K}(G) \neq \gamma_{2}(G)$, then every element of $\gamma_{2}(G)$ can be written as a product of at most two elements from $\mathrm{K}(G)$.

Along with the known results in the literature (as mentioned above), the preceding theorem provides a characterization of finite $p$-groups, $p \geq 5$, with $\gamma_{2}(G)$ of order $p^{4}$ such that each element of $\gamma_{2}(G)$ is a commutator. A classification of $p$-groups of order $p^{6}, p \geq 3$, with $\mathrm{K}(G)=$ $\gamma_{2}(G)$ is evident.

We now turn our attention to 2-groups. It is known that $\mathrm{K}(G)=\gamma_{2}(G)$ for all 2-groups $G$ of order at most $2^{6}$ [15]. The following theorem provides a characterization (upto isoclinism) of finite 2-groups $G$ with $\gamma_{2}(G)$ elementary abelian 2-group of order 16 such that $\mathrm{K}(G) \neq \gamma_{2}(G)$.

Theorem B. Let $G$ be a finite 2-group such that $\mathrm{Z}(G) \leq \gamma_{2}(G)$ ) and $\gamma_{2}(G)$ is elementary abelian of order 16 . Then $\mathrm{K}(G) \neq \gamma_{2}(G)$ if and only if one of the following holds true:

(1) $G$ is isoclinic to the following special 2-group of order $2^{9}$ presented as:

$$
\begin{aligned}
& \left\langle v_{1}, v_{2}, v_{3}, v_{4}, v_{5}\right|\left[v_{1}, v_{2}\right]=\left[v_{2}, v_{3}\right]=\left[v_{3}, v_{1}\right]=\left[v_{4}, v_{2}\right]=\left[v_{5}, v_{1}\right]=1,\left[v_{3}, v_{4}\right]=\left[v_{3}, v_{5}\right], \\
& \left.[x, y, z]=1 \text { for all } x, y, z \in\left\{v_{1}, \ldots, v_{5}\right\}, v_{i}^{2}=1(1 \leq i \leq 5)\right\rangle .
\end{aligned}
$$

(2) $G$ is of order $p^{8}$ and nilpotency class 2 along with one of the following:

(2a) $G$ admits a non-central element whose centralizer in $G$ is a maximal subgroup.

(2b) $G$ is of conjugate type $\{1,4,8\}$ and admits no generating set $\left\{x_{1}, x_{2}, x_{3}, x_{4}\right\}$ such that $\left[x_{1}, x_{2}\right]=1=\left[x_{3}, x_{4}\right]$.

Moreover, if $\mathrm{K}(G) \neq \gamma_{2}(G)$, then every element of $\gamma_{2}(G)$ can be written as a product of at most two elements from $\mathrm{K}(G)$.

A GAP computation shows that there are 2917 groups $G$ of order $2^{8}$ with $\gamma_{2}(G)$ elementary abelian of order 16, out of which 1542 groups satisfy $\mathrm{K}(G)=\gamma_{2}(G)$.

Our notations for objects associated with a finite multiplicative group $G$ are mostly standard. If $x, y \in G$, then $x^{y}$ denotes the conjugate element $y^{-1} x y \in G$ and $[x, y]=[x, y]_{G}$ denotes the commutator $x^{-1} y^{-1} x y=x^{-1} x^{y} \in G$. If $x \in G$, then $[x, G]$ denotes the set $\{[x, g] \mid g \in G\}$ (and not the subgroup generated by these commutators). If $[x, G] \subseteq \mathrm{Z}(G)$, then $[x, G]$ becomes a subgroup of $G$. For $n \geq 1$, we define $\left[x,_{n+1} y\right]=\left[\left[x,{ }_{n} y\right], y\right]$, where $\left[x,{ }_{1} y\right]=[x, y]$. For a subgroup $H$ of $G, \mathrm{C}_{G}(H)$ denotes the centralizer of $H$ in $G$ and for an element $x \in G, \mathrm{C}_{G}(x)$ denotes the centralizer of $x$ in $G$. By $\mathbb{F}_{p}$ we denote the field of $p$ elements. We write the subgroups in the lower central series of $G$ as $\gamma_{n}(G)$, where $n$ runs over all strictly positive integers. For a finite $p$-group $G$, we define $\Omega_{i}(G):=\left\langle x \in G \mid x^{p^{i}}=1\right\rangle$ and $\mho_{i}(G)=\left\langle x^{p^{i}} \mid x \in G\right\rangle$. 
In Section 2 we present some preliminaries and a reduction argument. Sections 4, 5 and 6 deal with $p$-groups under consideration having nilpotency class 2, 4 and 3, respectively. Proof of Theorem A is presented in Section 6. 2-groups under consideration are dealt with in Section 7, where a proof of Theorem B is presented. Several examples are exhibited in Section 8. We conclude this section with the remark that we used Magma [1] and GAP [3] for establishing our results for small primes, before writing final proofs.

\section{Reductions}

We start with the following concept of isoclinism of groups, introduced by P. Hall [10].

Let $X$ be a group and $\bar{X}=X / \mathrm{Z}(X)$. Then commutation in $X$ gives a well defined map $a_{X}: \bar{X} \times \bar{X} \mapsto \gamma_{2}(X)$ such that $a_{X}(x \mathrm{Z}(X), y \mathrm{Z}(X))=[x, y]$ for $(x, y) \in X \times X$. Two groups $G$ and $H$ are called isoclinic if there exists an isomorphism $\phi$ of the factor group $\bar{G}=G / \mathrm{Z}(G)$ onto $\bar{H}=H / \mathrm{Z}(H)$, and an isomorphism $\theta$ of the subgroup $\gamma_{2}(G)$ onto $\gamma_{2}(H)$ such that the following diagram is commutative

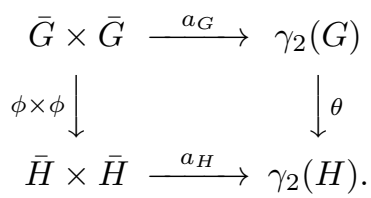

The resulting pair $(\phi, \theta)$ is called an isoclinism of $G$ onto $H$. Notice that isoclinism is an equivalence relation among groups.

Let $G$ be a finite $p$-group. Then it follows from [10] that there exists a finite $p$-group $H$ in the isoclinism family of $G$ such that $\mathrm{Z}(H) \leq \gamma_{2}(H)$. Such a group $H$ is called a stem group in the isoclinism family of $G$. As an easy consequence of this statement, we get

Corollary 2.1. A finite p-group $G$ with $\left|\gamma_{2}(G)\right|=p$ is isoclinic to an extraspecial p-group.

Lemma 2.2. Let $G$ and $H$ be two isoclinic finite p-groups. Then $\mathrm{K}(G)=\gamma_{2}(G)$ if and only if $\mathrm{K}(H)=\gamma_{2}(H)$.

Proof. Since isoclinism is an equivalence relation, it is sufficient to prove one side implication. So assume that $\mathrm{K}(G)=\gamma_{2}(G)$, and the map $a_{G}$ in the above commutative diagram is surjective. Let $u \in \gamma_{2}(H)$ be an arbitrary element. There exists $\left(\bar{g}_{1}, \bar{g}_{2}\right) \in \bar{G} \times \bar{G}$ such that $\theta\left(a_{G}\left(\bar{g}_{1}, \bar{g}_{2}\right)\right)=u$. Thus there exists $\left(\bar{h}_{1}, \bar{h}_{2}\right) \in \bar{H} \times \bar{H}$, namely $\left(\phi\left(\bar{g}_{1}\right), \phi\left(\bar{g}_{2}\right)\right)$, such that $a_{H}\left(\bar{h}_{1}, \bar{h}_{2}\right)=u$ proving that $a_{H}$ is surjective, and the proof is complete.

In view of the preceding lemma, it is sufficient to consider a stem group from a given isoclinism family.

Lemma 2.3. Let $G$ be a finite p-group such that $\gamma_{2}(G)$ is elementary abelian of order $p^{4}$. If the nilpotency class of $G$ is at most 3 , then $\left\{x^{p} \mid x \in G\right\} \subseteq \mathrm{Z}(G)$ for all $p \geq 3$. Otherwise the conclusion, in general, holds only for $p \geq 5$.

Proof. Since $\gamma_{2}(G)$ is of order $p^{4}$, the nilpotency class of $G$ is at most 5. Thus, $\gamma_{2}(G)$ being elementary abelian, for all $x, y \in G$, we have

$$
\left[x, y^{p}\right]=[x, y]^{p}[x, y, y]^{\left(\begin{array}{c}
p \\
2
\end{array}\right)}[x, y, y, y]^{\left(\begin{array}{c}
p \\
3
\end{array}\right)}[x, y, y, y, y]^{\left(\begin{array}{c}
p \\
4
\end{array}\right)}=1 .
$$


Hence $y^{p} \in Z(G)$.

Lemma 2.4. For a finite p-group $G$ of nilpotency class at least $4, \mathrm{Z}(G) \cap \gamma_{2}(G)$ can not be maximal in $\gamma_{2}(G)$.

Proof. Contrarily assume that $\mathrm{Z}(G) \cap \gamma_{2}(G)$ is maximal in $\gamma_{2}(G)$. Now consider the quotient group $\bar{G}:=G / \gamma_{4}(G)$, whose nilpotency class is 3 . Thus $\gamma_{2}(\bar{G}) \not \geq \mathrm{Z}(\bar{G})$. This means that $\mathrm{Z}(\bar{G})$ can not grow in $\gamma_{2}(\bar{G})=\gamma_{2}(G) / \gamma_{4}(G) . \bar{G}$ being of nilpotency class 3 , we have $\gamma_{3}(\bar{G}) \leq$ $\mathrm{Z}(\bar{G}) \cap \gamma_{2}(\bar{G})=\left(\mathrm{Z}(G) \gamma_{4}(G) / \gamma_{4}(G)\right) \cap \gamma_{2}(G) / \gamma_{4}(G)$, which is possible only when $\gamma_{3}(G) \leq \mathrm{Z}(G)$, a contradiction.

The proof of each of the following three lemmas is straightforward.

Lemma 2.5. Let $G$ be a finite group and $H \leq \gamma_{2}(G) \cap Z(G)$. If there exist $x_{1}, x_{2}, \ldots, x_{n}$ such that $\gamma_{2}(G) / H=\bigcup_{i=1}^{n}\left[x_{i} H, G / H\right]$ and $H \subseteq \bigcap_{i=1}^{n}\left[x_{i}, G\right]$, then $\gamma_{2}(G)=\bigcup_{i=1}^{n}\left[x_{i}, G\right]$.

Lemma 2.6. Let a finite group $G$ be a central product of its subgroups $M$ and $N$. Then $\mathrm{K}(G)=\gamma_{2}(G)$ if and only if $\mathrm{K}(M)=\gamma_{2}(M)$ and $\mathrm{K}(N)=\gamma_{2}(N)$.

Lemma 2.7. Let $G$ be a finite p-group and $H$ a normal subgroup of $G$ of order $p$ contained in $\gamma_{2}(G)$ such that $\gamma_{2}(G / H)=\mathrm{K}(G / H)$. Then every element of $\gamma_{2}(G)$ can be written as a product of at most two elements from $\mathrm{K}(G)$.

Following [4, p. 28], a group $G$ is said to be an amalgamated (internal) semidirect product of subgroups $H$ by $K$ over $M$, written $G=H \rtimes_{M} K$, if $H \unlhd G, G=H K$ and $H \cap K=M$. In particular, if $[H, K]=1$ then $M \leq Z(G)$, and we call $G$ the central product of $H$ by $K$ over $M$, written $G=H \times_{M} K$.

For a finite $p$-group $G$, the breadth of an element $x \in G$, denoted by $b(x)$, is defined as

$$
p^{b(x)}:=\left|G: \mathrm{C}_{G}(x)\right|,
$$

and the breadth of $G$, denoted by $b(G)$, is defined as

$$
b(G):=\max \{b(x) \mid x \in G\} .
$$

Remark 2.8. Let $G$ be a finite $p$-group with $\left|\gamma_{2}(G)\right|=p^{4}$. Then by [19, Theorem A] it follows that $b(G) \geq 3$. We'll use this information throughout without any further reference.

We'll need the following result of Rodney [21, Theorem B].

Theorem 2.9. Let $G$ be a finite group such that $\gamma_{2}(G)$ is elementary abelian of order $p^{3}$, where $p$ is any prime integer. Then $\mathrm{K}(G)=\gamma_{2}(G)$.

Our reduction arguments heavily depend on the following result of Parmeggiani and Stellmacher [19, Corollary]:

Theorem 2.10. Let $p$ be an odd prime and $G$ a finite p-group. Then $b(G)=3$ if and only if one of the following holds:

(i) $\left|\gamma_{2}(G)\right|=p^{3}$ and $|G: \mathrm{Z}(G)| \geq p^{4}$. 
(ii) $|G: \mathrm{Z}(G)|=p^{4}$ and $\left|\gamma_{2}(G)\right| \geq p^{4}$.

(iii) $\left|\gamma_{2}(G)\right|=p^{4}$ and there exists a normal subgroup $H$ of $G$ with $|H|=p$ and $\mid G / H$ : $\mathrm{Z}(G / H) \mid=p^{3}$.

We remark that the preceding result was also proved in [6, Corollary 3] for $p \geq 5$.

The following is the key result which reduces our study mainly to the groups of small orders.

Theorem 2.11. Let $L$ be a finite p-group such that $\mathrm{Z}(L) \leq \gamma_{2}(L), \gamma_{2}(L)$ is elementary abelian of order $p^{4}$ and $b(L)=3$. If the nilpotency class of $L$ is 3 and $p \geq 3$, then one of the following holds:

(i) There exists a 2-generator subgroup $G$ of $L$ having the same nilpotency class as that of $L$ such that $\gamma_{2}(G)=\gamma_{2}(L)$. Moreover, $|G|=p^{6}$, and if $|L| \geq p^{7}$, then $L$ is an amalgamated semidirect product of $G$ and a subgroup $K$ with $\left|\gamma_{2}(K)\right| \leq p$. Moreover, if $K$ is non-abelian, then it is isoclinic to an extraspecial p-group.

(ii) There exists a 2-generator subgroup $G$ of $L$ having the same nilpotency class as that of $L$ such that $\gamma_{2}(G)<\gamma_{2}(L)$. Moreover, $|G|=p^{5},|L| \geq p^{7}$ and $L$ is a central product of $G$ and a subgroup $K$ of nilpotency class 2 , which is isoclinic to an extraspecial p-group.

(iii) There exists a 3-generator subgroup $G$ of $L$ having the same nilpotency class as that of $L$ such that $\gamma_{2}(G)=\gamma_{2}(L)$. Moreover, $|G|=p^{7}$, and if $|L| \geq p^{8}$, then $L$ is an amalgamated semidirect product of $G$ and a subgroup $K$ with $\left|\gamma_{2}(K)\right| \leq p$. Moreover, if $K$ is non-abelian, then it is isoclinic to an extraspecial p-group.

If the nilpotency class of $L$ is 4 and $p \geq 3$, then only (i) holds.

Proof. Since $b(L)=3$, it follows from Theorem 2.10 that either $|L: \mathrm{Z}(L)|=p^{4}$ or $L$ admits a subgroup $H$ of order $p$ such that $|L / H: \mathrm{Z}(L / H)|=p^{3}$. If $|L: \mathrm{Z}(L)|=p^{4}$, then it follows from Lemma 2.3 that $L$ itself is either a 2-generator group of order $p^{6}$ or a 3-generator group of order $p^{7}$. For, $p^{2} \leq|\mathrm{Z}(L)| \leq p^{3}$; otherwise the nilpotency class of $L$ will at most be 2 , which we are not considering. Moreover, when $L$ is a 3 -generator group of order $p^{7}$, then it follows from Lemma 2.4 that the nilpotency class of $L$ is 3 . Now consider the second case, which we divide into two subcases, depending on the nilpotency class of $L$.

First assume that the nilpotency class of $L$ is 3 . Then $H=\gamma_{3}(L), H<\gamma_{3}(L)$ or $H \not \leq \gamma_{3}(L)$. We consider these possibilities one by one. If $H=\gamma_{3}(L)$, then the nilpotency class of $L / H$ is 2 and $|L / H: \mathrm{Z}(L / H)|=p^{3}$, and therefore, using Lemma 2.3, it follows that except three generators $a, b, c$ (say) of $L$, all other generators $x_{1}, x_{2}, \ldots, x_{k}, k \geq 0$, are such that $\left[x_{i}, L\right] \leq H$. Since $\mathrm{Z}(L) \leq \gamma_{2}(L)$, we can, more precisely, say that $\left[x_{i}, L\right]=H$ for all $1 \leq i \leq k$. So it follows that $\gamma_{2}(L) / H=\gamma_{2}(G) H / H$ is of order $p^{3}$, where $G:=\langle a, b, c\rangle$ is a subgroup of $L$. We claim that $H \leq \gamma_{2}(G)$. As observed above $\left[x_{i}, L\right]=H$ is of order $p$, it follows that, for all $1 \leq i \leq k$, $\mathrm{C}_{L}\left(x_{i}\right)$ is maximal in $L$, and therefore contains $\gamma_{2}(L)$. Thus any generator $h$ of $H$, which lies in $\gamma_{3}(L)=\left[\gamma_{2}(L), L\right]$, can be written as

$$
h=\left[w_{1}^{\alpha_{1}} w_{2}^{\alpha_{2}} w_{3}^{\alpha_{3}}, a^{\beta_{1}} b^{\beta_{2}} c^{\beta_{3}}\right] \in \gamma_{3}(G),
$$

where $w_{1} H, w_{2} H, w_{3} H$ generate $\gamma_{2}(G) H / H$. The nice presentation of $h$ in the preceding statement is possible because $H \leq \mathrm{Z}(L)$. Hence our claim follows, which, in turn, implies that $\gamma_{2}(G)=\gamma_{2}(L)$. Thus $G$ is of order $p^{7}$ and nilpotency class 3 . Let $K:=\left\langle x_{1}, \ldots, x_{k}\right\rangle$ be a subgroup of $L$. Since $\left[x_{i}, L\right]=H$ for $1 \leq i \leq k$, we have $\gamma_{2}(K) \leq H$, which shows that the 
nilpotency class of $K$ is at most 2. Since $\gamma_{2}(L) \leq G, K$ acts on $G$ by conjugation. Hence $L$ takes the desired form. If $K$ is non-abelian, then, in view of Corollary 2.1, $K$ is isoclinic to an extraspecial $p$-group.

If $H<\gamma_{3}(L)$, then the nilpotency class of $L / H$ is 3 and $|L / H: \mathrm{Z}(L / H)|=p^{3}$. Hence by the given hypothesis and Lemma 2.3 , we conclude that $L$ can be generated by $\left\{a, b, x_{1}, \ldots, x_{k}\right\}$ such that $\left[x_{i}, L\right]=H$. Now, using the same arguments as in the preceding case, the assertion follows by assuming $G:=\langle a, b\rangle$ and $K:=\left\langle x_{1}, \ldots, x_{k}\right\rangle$, where $|G|=p^{6}$. Finally, if $H \not \leq \gamma_{3}(L)$, then, obviously, $H \cap \gamma_{3}(L)=1$. Thus the nilpotency class of $L / H$ is also 3. Again invoking the given hypothesis and Lemma 2.3, we can assume that $L$ is generated by the set $\left\{a, b, x_{1}, \ldots, x_{k}\right\}$ such that $\left[x_{i}, L\right]=H$. Let $G_{1}:=\langle a, b\rangle$. Notice that $\gamma_{2}\left(G_{1}\right) H / H=\gamma_{2}(L) / H$ is of order $p^{3},\left|G_{1} H / H\right|=p^{5}$, and $G_{1}$ and $L$ agree on the nilpotency class. Since $G_{1}$ is 2-generator, $\gamma_{2}\left(G_{1}\right) / \gamma_{3}\left(G_{1}\right)$ is cyclic (of order $p$ ). Thus $G_{1}$ can not contain $H$, which implies that $\left|G_{1}\right|=p^{5}$. If $G_{1} \leq \mathrm{C}_{L}\left(x_{i}\right)$, for all $1 \leq i \leq k$, then $K:=\left\langle x_{1}, \ldots, x_{k}\right\rangle$ with $\gamma_{2}(K)=H$ is isoclinic to an extraspecial $p$-group, and therefore $L$ is a central product of $G_{1}$ and $K$ amalgamating some subgroup (possibly trivial). Hence $G=G_{1}$ and $K$ are the desired subgroups. So assume that $\left[x_{i}, G_{1}\right] \neq 1$ for some $i$. Thus $\left[x_{i}, G_{1}\right]=H$, and the subgroup $G:=\left\langle a, b, x_{i}\right\rangle$ of $L$ is of order $p^{7}$. Hence, as argued above, one can easily see that $G$ and $K:=\left\langle x_{1}, \ldots, x_{i-1}, x_{i+1}, \ldots, x_{k}\right\rangle$ are the desired subgroups of $L$.

We now assume that the nilpotency class of $L$ is 4 . Then either $H=\gamma_{4}(L)$ or $H \neq \gamma_{4}(L)$. We claim that there is no $L$ of nilptency class 4 such that $H \neq \gamma_{4}(L)$. If such an $L$ exists, then the nilpotency class of $L / H$ is 4 , which is not possible as $(L / H) / \mathrm{Z}(L / H)$, being of order $p^{3}$, can have nilpotency class at most 2. So assume that $H=\gamma_{4}(L)$. Then the nilpotency class of $L / H$ is 3 . Hence, by the given hypothesis, we conclude that $L$ can be generated by $\left\{a, b, x_{1}, \ldots, x_{k}\right\}$ such that $\left[x_{i}, L\right]=H$. Since $|L / H: \mathrm{Z}(L / H)|=p^{3}$, it follows that $a^{p}, b^{p} \in \gamma_{2}(L)$. Now, using the same arguments as above, the assertion follows by assuming $G:=\langle a, b\rangle$ and $K:=\left\langle x_{1}, \ldots, x_{k}\right\rangle$, where $|G|=p^{6}$. This completes the proof of the theorem.

\section{GROUPS OF CLASS 2}

This section is devoted to the investigation of the question under consideration for groups of class 2 . We say that a finite $p$-group $G$ is of conjugate type $\left\{1, p^{r}, p^{s}\right\}$ if this set constitutes the set of conjugacy class sizes of all elements of $G$, where $r \leq s$ are positive integers.

Lemma 3.1. Let $G$ be a finite p-group of order $p^{8}$ and nilpotency class 2 such that $\gamma_{2}(G)$ is elementary abelian of order $p^{4}$. If $G$ is not of conjugate type $\left\{1, p^{3}\right\}$ or $\left\{1, p^{2}, p^{3}\right\}$, then $\mathrm{K}(G) \neq G^{\prime}$. Moreover, every element of $\gamma_{2}(G)$ can be written as a product of at most two elements from $\mathrm{K}(G)$.

Proof. It follows by the given hypothesis that $\mathrm{Z}(G)=\gamma_{2}(G)$ and $G$ is minimally generated by 4 elements. Notice that $b(G)=3$. Again by the given hypothesis there exists an element $d \in G-\gamma_{2}(G)$ such that $\mathrm{C}_{G}(d)$ is maximal in $G$. We can always extend $\{d\}$ to a generating set $\{a, b, c, d\}$ for $G$ such that $\gamma_{2}(G)=\langle[a, b],[a, c],[b, c],[c, d]\rangle$. We claim that $[a, b][c, d]$ is not in $\mathrm{K}(G)$. Contrarily assume that $[a, b][c, d] \in \mathrm{K}(G)$. Thus

$$
[a, b][c, d]=\left[a^{\alpha_{1}} b^{\alpha_{2}} c^{\alpha_{3}} d^{\alpha_{4}}, a^{\beta_{1}} b^{\beta_{2}} c^{\beta_{3}} d^{\beta_{4}}\right],
$$


where $\alpha_{i}, \beta_{j} \in \mathbb{F}_{p}$ for $1 \leq i, j \leq 4$. Expanding the right hand side and comparing the powers of the generators of $\gamma_{2}(G)$, we get the following set of equations:

$$
\begin{aligned}
& \beta_{2} \alpha_{1}-\alpha_{2} \beta_{1}=1, \\
& \beta_{3} \alpha_{2}-\alpha_{3} \beta_{2}=0, \\
& \beta_{3} \alpha_{1}-\alpha_{3} \beta_{1}=0 \\
& \beta_{4} \alpha_{3}-\alpha_{4} \beta_{3}=1 .
\end{aligned}
$$

First assume that $\beta_{3} \neq 0$. Then from (3.2) and (3.3), we get $\alpha_{2}=\alpha_{3} \beta_{2} \beta_{3}{ }^{-1}$ and $\alpha_{1}=$ $\alpha_{3} \beta_{1} \beta_{3}{ }^{-1}$. Notice that these values of $\alpha_{1}$ and $\alpha_{2}$ contradict (3.1). Thus $\beta_{3}=0$. That $\alpha_{3} \neq 0$ follows from (3.4) after inserting $\beta_{3}=0$. This then implies, along with (3.2) and (3.3), that $\beta_{1}=\beta_{2}=0$, which contradicts (3.1). Hence the above system of equations has no solution, which settles our claim.

Let $H$ be any subgroup of $\gamma_{2}(G)$ of order $p$. Then it follows from Theorem 2.9 that $\gamma_{2}(G / H)=$ $\mathrm{K}(G / H)$. Hence the proof is complete by Lemma 2.7

Lemma 3.2. Let $G$ be a finite p-group of order $p^{8}$, nilpotency class 2 and conjugate type $\left\{1, p^{2}, p^{3}\right\}$ such that $\gamma_{2}(G)$ is elementary abelian of order $p^{4}$. Then $\mathrm{K}(G)=\gamma_{2}(G)$ if and only if $G$ admits a generating set $\{a, b, c, d\}$ such that $[a, b]=1=[c, d]$. Moreover, if $\mathrm{K}(G) \neq \gamma_{2}(G)$, then every element of $\gamma_{2}(G)$ can be written as a product of at most two elements from $\mathrm{K}(G)$.

Proof. Let $G$ admit a generating set $\{a, b, c, d\}$ such that $[a, b]=1=[c, d]$. Then $\gamma_{2}(G)=$ $\langle[a, c],[b, c],[a, d],[b, d]\rangle$. Given $\varepsilon, \lambda, \mu, \eta \in \mathbb{F}_{p}$, we are going to show that

$$
[a, c]^{\varepsilon}[b, c]^{\lambda}[a, d]^{\mu}[b, d]^{\eta}=\left[a^{\alpha_{1}} b^{\alpha_{2}} c, a^{\beta_{1}} b^{\beta_{2}} d^{\beta_{4}}\right]
$$

for some $\alpha_{i}, \beta_{j} \in \mathbb{F}_{p}$. We go in the reverse way. Expanding the right hand side of (3.5) and comparing powers on both sides, we get

$$
\beta_{1}=-\varepsilon, \beta_{2}=-\lambda, \alpha_{1} \beta_{4}=\mu, \alpha_{2} \beta_{4}=\eta .
$$

It is easy to see that for given $\varepsilon, \lambda, \mu, \eta \in \mathbb{F}_{p}$, we can always find some $\alpha_{i}, \beta_{j} \in \mathbb{F}_{p}$ such that (3.5) holds true. Hence $\mathrm{K}(G)=\gamma_{2}(G)$.

We provide a contrapositive proof of the converse part. We assume that $G$ admits no generating set $\left\{x_{1}, x_{2}, x_{3}, x_{4}\right\}$ such that $\left[x_{1}, x_{2}\right]=1=\left[x_{3}, x_{4}\right]$. By the given hypothesis, we can always choose a generating set $\{a, b, c, d\}$ for $G$ such that $[a, c]=1$ and none of the other basic commutators of weight two in generators is trivial. So we can assume that

$$
[c, d]=[a, b]^{t_{1}}[b, c]^{t_{2}}[b, d]^{t_{3}}[a, d]^{t_{4}},
$$

where $t_{i} \in \mathbb{F}_{p}$ and $1 \leq i \leq 4$, and therefore $\gamma_{2}(G)=\langle[a, b],[b, c],[b, d],[a, d]\rangle$.

We can write the preceding equation as

$$
\left[b, a^{-t_{1}} c^{t_{2}} d^{t_{3}}\right]\left[d, a^{-t_{4}} c\right]=1 .
$$

We claim that $t_{3}=0$. If $t_{3} \neq 0$, then replacing $d$ by $d^{\prime}:=a^{-t_{1}} c^{t_{2}} d^{t_{3}}$ and a proper substitution reduces (3.6) to $\left[d^{\prime}, b^{-1} a^{-t_{4} t_{3}^{-1}} c^{t_{3}^{-1}}\right]=1$. Now replacing $b$ by $b^{\prime}:=b^{-1} a^{-t_{4} t_{3}^{-1}} c^{t_{3}^{-1}}$, we get a generating set $\left\{a, b^{\prime}, c, d^{\prime}\right\}$ for $G$ such that $\gamma_{2}(G)=\left\langle\left[a, b^{\prime}\right],\left[b^{\prime}, c\right],\left[b^{\prime}, d^{\prime}\right],\left[c, d^{\prime}\right]\right\rangle$ and $[a, c]=1=$ $\left[b^{\prime}, d^{\prime}\right]$, which contradicts our hypothesis. Our claim is now settled. 
So now onwards we assume that $t_{3}=0$. Hence (3.6) reduces to $\left[b, a^{-t_{1}} c^{t_{2}}\right]\left[d, c a^{-t_{4}}\right]=1$. Now replace $c$ by $c^{\prime}:=c a^{-t_{4}}$. A simple computation gives

$$
\left[b, a^{-t_{1}} c^{t_{2}}\right]=\left[b, a^{-t_{1}}\left(c^{\prime} a^{t_{4}}\right)^{t_{2}}\right]=\left[b, a^{t_{4} t_{2}-t_{1}} c^{\prime t_{2}}\right] .
$$

Thus (3.6) reduces to

$$
\left[b, a^{t_{4} t_{2}-t_{1}} c^{\prime t_{2}}\right]\left[d, c^{\prime}\right]=1 .
$$

We claim that $t_{4} t_{2}-t_{1} \neq 0$. Contrarily assume that $t_{4} t_{2}-t_{1}=0$. Then (3.7) takes the form $\left[b, c^{\prime t_{2}}\right]\left[d, c^{\prime}\right]=1$, which gives $\left[b^{t_{2}} d, c^{\prime}\right]=1$. Now replacing $d$ by $d^{\prime}:=b^{t_{2}} d$, we get a generating set $\left\{a, b, c^{\prime}, d^{\prime}\right\}$ of $G$ such that $\left[a, c^{\prime}\right]=1=\left[d^{\prime}, c^{\prime}\right]$, which gives that the size of the conjugacy class of $c^{\prime}$ in $G$ is $p$, a contradiction to the given hypothesis. This settles our claim.

So we now assume $t_{4} t_{2}-t_{1} \neq 0$. Then replacing $a$ by $a^{\prime}:=a^{t_{4} t_{2}-t_{1}} c^{\prime t_{2}}$, we get a new generating set $\left\{a^{\prime}, b, c^{\prime}, d\right\}$ such that $\gamma_{2}(G)=\left\langle\left[a^{\prime}, b\right],\left[b, c^{\prime}\right],[b, d],\left[a^{\prime}, d\right]\right\rangle,\left[a^{\prime}, c^{\prime}\right]=1$ and, by (3.7), $\left[a^{\prime}, b\right]^{-1}=\left[c^{\prime}, d\right]$. Now we claim that $\left[b, c^{\prime}\right]^{\lambda}\left[a^{\prime}, d\right]^{\mu} \notin \mathrm{K}(G)$ for some $\lambda, \mu \in \mathbb{F}_{p}^{*}$. Contrarily assume that $\left[b, c^{\prime}\right]^{\lambda}\left[a^{\prime}, d\right]^{\mu} \in \mathrm{K}(G)$ for all $\lambda, \mu \in \mathbb{F}_{p}^{*}$. Thus

$$
\left[b, c^{\prime}\right]^{\lambda}\left[a^{\prime}, d\right]^{\mu}=\left[a^{\prime \alpha_{1}} b^{\alpha_{2}} c^{\prime \alpha_{3}} d^{\alpha_{4}}, a^{\prime \beta_{1}} b^{\beta_{2}} c^{\prime \beta_{3}} d^{\beta_{4}}\right],
$$

where $\alpha_{i}, \beta_{j} \in \mathbb{F}_{p}$ for $1 \leq i, j \leq 4$. Expanding the right hand side and comparing powers both side, we get

$$
\begin{aligned}
\alpha_{1} \beta_{4}-\alpha_{4} \beta_{1} & =\mu, \\
\alpha_{2} \beta_{4}-\alpha_{4} \beta_{2} & =0, \\
\alpha_{2} \beta_{3}-\alpha_{3} \beta_{2} & =\lambda, \\
\alpha_{1} \beta_{2}-\alpha_{2} \beta_{1}-\alpha_{3} \beta_{4}+\alpha_{4} \beta_{3} & =0 .
\end{aligned}
$$

First assume that $\alpha_{2}=0$. Then from (3.10) $\alpha_{3} \beta_{2}=-\lambda$, therefore by (3.9) $\alpha_{4}=0$. Now by (3.8) $\alpha_{1} \beta_{4}=\mu$. Since $\alpha_{1}$ and $\alpha_{3}$ are non-zero, by substituting the value of $\beta_{2}=-\lambda \alpha_{3}^{-1}$ and $\beta_{4}=\mu \alpha_{1}^{-1}$ in (3.11), we get $\lambda \alpha_{1} \alpha_{3}^{-1}+\mu \alpha_{3} \alpha_{1}^{-1}=0$. Thus $\left(\alpha_{1} \alpha_{3}^{-1}\right)^{2}=-\lambda^{-1} \mu$, which is a contradiction because we can choose $\lambda, \mu \in \mathbb{F}_{p}^{*}$ such that $-\lambda^{-1} \mu$ is non square. Now we assume that $\alpha_{2} \neq 0$. If $\alpha_{4}=0$, then (3.9) implies that $\beta_{4}=0$, which contradicts (3.8). So finally assume that both $\alpha_{2}$ and $\alpha_{4}$ are non zero. The augmented matrix of above system of equations, with $\beta$ 's as variables, is given by

Performing row operations

$$
M=\left[\begin{array}{ccccc}
-\alpha_{4} & 0 & 0 & \alpha_{1} & \mu \\
0 & -\alpha_{4} & 0 & \alpha_{2} & 0 \\
0 & -\alpha_{3} & \alpha_{2} & 0 & \lambda \\
-\alpha_{2} & \alpha_{1} & \alpha_{4} & -\alpha_{3} & 0
\end{array}\right]
$$

(i) $R_{1} \rightarrow-\alpha_{4}^{-1} R_{1}, R_{2} \rightarrow-\alpha_{4}^{-1} R_{2}$,

(ii) $R_{4} \rightarrow R_{4}+\alpha_{2} R_{1}, R_{3} \rightarrow R_{3}+\alpha_{3} R_{2}$,

(iii) $R_{3} \rightarrow \alpha_{2}^{-1} R_{3}, R_{4} \rightarrow R_{4}-\alpha_{1} R_{2}$,

(iv) $R_{4} \rightarrow R_{4}-\alpha_{4} R_{3}$,

we get

$$
M_{1}=\left[\begin{array}{ccccc}
1 & 0 & 0 & -\alpha_{1} \alpha_{4}^{-1} & -\mu \alpha_{4}^{-1} \\
0 & 1 & 0 & -\alpha_{2} \alpha_{4}^{-1} & 0 \\
0 & 0 & 1 & -\alpha_{3} \alpha_{4}^{-1} & \lambda \alpha_{2}^{-1} \\
0 & 0 & 0 & 0 & -\mu \alpha_{2} \alpha_{4}^{-1}-\lambda \alpha_{2}^{-1} \alpha_{4}
\end{array}\right] .
$$


If the above system of equations admits a solution, then $\mu \alpha_{2} \alpha_{4}^{-1}+\lambda \alpha_{2}^{-1} \alpha_{4}=0$. This gives $\left(\alpha_{2} \alpha_{4}^{-1}\right)^{2}=-\lambda \mu^{-1}$, which is a contradiction again, because we can choose $\lambda, \mu \in \mathbb{F}_{p}^{*}$ such that $-\lambda \mu^{-1}$ is non square. The final assertion holds by taking $G / H$, where $H$ is any subgroup of $\gamma_{2}(G)$ of order $p$, and then using Theorem 2.9 and Lemma 2.7. The proof of the lemma is now complete.

Lemma 3.3. Let $G$ be a finite p-group of order $p^{8}$, nilpotency class 2 and conjugate type $\left\{1, p^{3}\right\}$ such that $\gamma_{2}(G)$ is elementary abelian of order $p^{4}$. Then $\mathrm{K}(G)=\gamma_{2}(G)$.

Proof. Using [18, Lemma 3.14], we can assume that the exponent of $G$ is $p$. Now, it follows from [18, Theorem 1.2] that $G$ has the following presentation:

$$
G=\left\langle a, b, c, d \mid a^{p}=b^{p}=c^{p}=d^{p}=1,[c, d]=[a, b]^{-1},[a, c]=[b, d]^{-r},[[x, y], z]=1\right\rangle
$$

where $x, y, z \in\{a, b, c, d\}$ and $r$ is any non-quadratic residue mod $p$. We'll show that for given $\lambda, \mu, \nu, \xi \in \mathbb{F}_{p}$, there exist $\alpha_{i}, \beta_{i} \in \mathbb{F}_{p}$ such that

$$
[a, b]^{\lambda}[b, c]^{\mu}[b, d]^{\nu}[a, d]^{\xi}=\left[a^{\alpha_{1}} b^{\alpha_{2}} c^{\alpha_{3}}, a^{\beta_{1}} b^{\beta_{2}} c^{\beta_{3}} d^{\beta_{4}}\right] .
$$

Solving both sides of the preceding equation and comparing the powers, we get

$$
\begin{aligned}
\alpha_{1} \beta_{2}-\alpha_{2} \beta_{1}-\alpha_{3} \beta_{4} & =\lambda, \\
\alpha_{2} \beta_{3}-\alpha_{3} \beta_{2} & =\mu, \\
\alpha_{2} \beta_{4}-r\left(\alpha_{1} \beta_{3}-\alpha_{3} \beta_{1}\right) & =\nu, \\
\alpha_{1} \beta_{4} & =\xi .
\end{aligned}
$$

It is sufficient to show that this system of equations, $\beta_{i}$ 's as variables, admits a solution. The augmented matrix for this system of equations is as follows:

$$
M=\left[\begin{array}{ccccc}
-\alpha_{2} & \alpha_{1} & 0 & -\alpha_{3} & \lambda \\
0 & -\alpha_{3} & \alpha_{2} & 0 & \mu \\
r \alpha_{3} & 0 & -r \alpha_{1} & \alpha_{2} & \nu \\
0 & 0 & 0 & \alpha_{1} & \xi
\end{array}\right]
$$

Performing row operations

(i) $R_{3} \rightarrow \alpha_{2} R_{3}$, (ii) $R_{3} \rightarrow R_{3}+r \alpha_{3} R_{1}$ and (iii) $R_{3} \rightarrow R_{3}+r \alpha_{1} R_{2}$, the above matrix transforms to

$$
M_{1}=\left[\begin{array}{ccccc}
-\alpha_{2} & \alpha_{1} & 0 & -\alpha_{3} & \lambda \\
0 & -\alpha_{3} & \alpha_{2} & 0 & \mu \\
0 & 0 & 0 & \alpha_{2}^{2}-r \alpha_{3}^{2} & \nu \alpha_{2}+r \lambda \alpha_{3}+r \mu \alpha_{1} \\
0 & 0 & 0 & \alpha_{1} & \xi
\end{array}\right] .
$$

It is easy to see that the above system of equations admits a solution only if

$$
\xi\left(\alpha_{2}^{2}-r \alpha_{3}^{2}\right)-\alpha_{1}\left(\nu \alpha_{2}+r \lambda \alpha_{3}+r \mu \alpha_{1}\right)=0,
$$

or equivalenty

$$
r \mu \alpha_{1}^{2}+\left(\nu \alpha_{2}+r \lambda \alpha_{3}\right) \alpha_{1}-\xi\left(\alpha_{2}^{2}-r \alpha_{3}^{2}\right)=0 .
$$

Viewing the preceding equation as a quadratic equation in $\alpha_{1}$, notice that it has a solution in $\mathbb{F}_{p}$ if its discriminant is zero or a quadratic residue $\bmod p$. The discriminant, after an easy computation, takes the form

$$
\left(\nu^{2}+4 r \mu \xi\right) \alpha_{2}^{2}+2 r \lambda \nu \alpha_{2} \alpha_{3}+r^{2}\left(\lambda^{2}-4 \mu \xi\right) \alpha_{3}^{2} .
$$


Notice that (3.16) is of the form $f \alpha_{2}^{2}+g \alpha_{2} \alpha_{3}+h \alpha_{3}^{2}$, where $f, g, h \in \mathbb{F}_{p}$. As we know, there exist $\alpha_{2}, \alpha_{3} \in \mathbb{F}_{p}$ such that (3.16) is either zero or a quadratic residue $\bmod p$. This completes the proof.

Lemma 3.4. Let $G$ be a finite p-group of nilpotency class 2 and order at least $p^{9}, p \geq 3$, such that $\gamma_{2}(G)$ is elementary abelian of order $p^{4}$ and $\mathrm{Z}(G)=\gamma_{2}(G)$. Then $\mathrm{K}(G)=\gamma_{2}(G)$.

Proof. If $b(G)=4$, then we are done. So assume that $b(G)=3$. Theorem 2.10 now guarantees the existence of a normal subgroup $H$ of $G$ of order $p$ such that $|G / H: \mathrm{Z}(G / H)|=p^{3}$. Thus, using the fact that the exponent of $G / \mathrm{Z}(G)$ is $p$ (which follows from the given hypothesis), we can assume that

$$
G=\left\langle a, b, c, x_{1}, \ldots, x_{k}\right\rangle,
$$

where $k \geq 2$ and $\left[x_{i}, G\right]=H$ for $1 \leq i \leq k$. Let $S:=\langle a, b, c\rangle$ be the subgroup of $G$ generated by $a, b, c$. Notice that $|S|=p^{6}$ and $\left|\gamma_{2}(S)\right|=p^{3}$, and therefore $\mathrm{K}(S)=\gamma_{2}(S)$. If $S \leq \mathrm{C}_{G}\left(x_{i}\right)$ for all $1 \leq i \leq k$, then, since $\mathrm{Z}(G)=\gamma_{2}(G), G$ can written as a central product of $S$ and a $k$-generator group isoclinic to an extraspecial $p$-groups generated by $\left\{x_{1}, \ldots, x_{k}\right\}$. Now using Lemma 2.6. we have $\mathrm{K}(G)=\gamma_{2}(G)$. So assume that $\left[x_{t}, S\right]=H$ for some $t \in\{1, \ldots, k\}$. By reordering the set $\left\{x_{1}, \ldots, x_{k}\right\}$, if necessary, we can assume that $t=1$. For simplicity of notation, we set $d:=x_{1}$. Since $\mathrm{C}_{G}(d)$ is a maximal subgroup of $G$, we can modify the generators $a, b, c$ such that $\gamma_{2}(G)=\langle[a, b],[a, c],[b, c],[c, d]\rangle,[a, d]=[b, d]=1$ and $H=\langle[c, d]\rangle$. We can also assume, by suitable modification of $x_{i}$, that $\left[c, x_{i}\right]=1$ for all $2 \leq i \leq k$.

Let $\alpha_{i} \in \mathbb{F}_{p}$ for $1 \leq i \leq 4$. If $\alpha_{3} \neq 0$, then the following identity holds:

$$
[a, b]^{\alpha_{1}}[b, c]^{\alpha_{2}}[a, c]^{\alpha_{3}}[c, d]^{\alpha_{4}}=\left[c b^{\alpha_{1} \alpha_{3}{ }^{-1}}, a^{-\alpha_{3}} b^{-\alpha_{2}} d^{\alpha_{4}}\right] .
$$

Thus any element of $\gamma_{2}(G)$ involving $[a, c]$ is a commutator. If $\alpha_{3}=0$ and $\alpha_{2} \neq 0$, then for any $\alpha_{1}, \alpha_{4} \in \mathbb{F}_{p}$ we have

$$
[a, b]^{\alpha_{1}}[b, c]^{\alpha_{2}}[c, d]^{\alpha_{4}}=\left[b d^{-\alpha_{4} \alpha_{2}{ }^{-1}}, a^{-\alpha_{1}} c^{\alpha_{2}}\right] .
$$

So, it only remains to show that elements of the form $[a, b]^{\alpha_{1}}[c, d]^{\alpha_{4}}$ are commutators, where both $\alpha_{1}$ and $\alpha_{4}$ are non-zero in $\mathbb{F}_{p}$.

If $\left[a, x_{i}\right]=[c, d]^{\beta}$ for some $2 \leq i \leq k$ and $\beta \in \mathbb{F}_{p}^{*}$, then

$$
[a, b]^{\alpha_{1}}[c, d]^{\alpha_{4}}=\left[a, b^{\alpha_{1}} x_{i}{ }^{\beta^{-1} \alpha_{4}}\right] .
$$

Set $A:=\left\langle x_{2}, \ldots, x_{k}\right\rangle$. Now suppose that $a \in \mathrm{C}_{G}(A)$, but $b \notin \mathrm{C}_{G}(A)$. So there must exist an $x_{i}$, $2 \leq i \leq k$, such that $\left[b, x_{i}\right]=[c, d]^{\beta}$, where $\beta \in \mathbb{F}_{p}^{*}$. Then

$$
[a, b]^{\alpha_{1}}[c, d]^{\alpha_{4}}=\left[b, a^{-\alpha_{1}} x_{i}^{\beta^{-1} \alpha_{4}}\right] .
$$

Let $a, b \in \mathrm{C}_{G}(A)$. If $\left[d, x_{i}\right]=[c, d]^{\beta}$ for some $2 \leq i \leq k$ and $\beta \in \mathbb{F}_{p}^{*}$, then

$$
[a, b]^{\alpha_{1}}[c, d]^{\alpha_{4}}=\left[a d, b^{\alpha_{1}} x_{i}^{\beta^{-1} \alpha_{4}}\right] .
$$

So finally assume that $a, b, c, d \in \mathrm{C}_{G}(A)$. Notice that, in this case, $k \geq 3$ and $\gamma_{2}(A)=H$, since $[A, G]=H$. Hence $\left[x_{i}, x_{j}\right]=[c, d]^{\beta}$ for some $2 \leq i, j \leq k$ and $\beta \in \mathbb{F}_{p}^{*}$, and therefore we have

$$
[a, b]^{\alpha_{1}}[c, d]^{\alpha_{4}}=\left[b x_{i}, a^{-\alpha_{1}} x_{j}^{\alpha_{4} \beta^{-1}}\right] .
$$

This shows that each element of $\gamma_{2}(G)$ is a commutator, and the proof is complete. 


\section{Groups of Class 4}

We start with groups of order $p^{6}$. Upto isoclinism, there are only 3 groups of order $p^{6}$ whose commutator subgroup is elementary abelian of order $p^{4}, p \geq 3$ (see [14]).

Lemma 4.1. Let $G$ be a group of order $p^{6}, p \geq 3$ with $\gamma_{2}(G)$ is elementary abelian of order $p^{4}$. Then $\mathrm{K}(G)=\gamma_{2}(G)$ if and only if $|\mathrm{Z}(G)|=p$. Moreover, if $\mathrm{K}(G) \neq \gamma_{2}(G)$, then every element of $\gamma_{2}(G)$ can be written as a product of at most two elements from $\mathrm{K}(G)$.

Proof. It follows from [14 that upto isoclinism there are only three groups $G$ of order $p^{6}, p \geq 3$, such that $\gamma_{2}(G)$ is elementary abelian of order $p^{4}$. These fall under isoclinism families $\phi_{23}, \phi_{40}$ and $\phi_{41}$. All these groups are of nilpotency class 4 . For any group $G$ belonging to $\phi_{23}$, we have $|\mathrm{Z}(G)|=p^{2}$ and it can be easily checked using GAP [3] or Magma [1] that $p \geq 5$. Let $G$ be a representative from $\phi_{23}$, which is presented as

$$
\begin{gathered}
G=\left\langle\alpha, \alpha_{1}, \alpha_{2}, \alpha_{3}, \alpha_{4}, \gamma\right|\left[\alpha_{i}, \alpha\right]=\alpha_{i+1},\left[\alpha_{1}, \alpha_{2}\right]=\gamma, \\
\left.\alpha^{p}=\gamma, \alpha_{1}{ }^{p}=\alpha_{i+1}^{p}=\gamma^{p}=1(i=1,2,3)\right\rangle .
\end{gathered}
$$

Notice that $G$ is minimally generated by $\alpha$ and $\alpha_{1}$, exponent of $\gamma_{2}(G)$ is $p$ and $\left[\alpha_{1},\left[\alpha, \alpha_{1}\right]\right]=$ $\gamma \in Z(G)$. Hence it follows from [15, Proposition 5.3] that $\mathrm{K}(G) \neq \gamma_{2}(G)$. More precisely, $\alpha_{4} \gamma \notin \mathrm{K}(G)$.

For all groups $G$ from the isoclinism families $\phi_{40}$ and $\phi_{41},|\mathrm{Z}(G)|=p$. If $p=3$, then an easy GAP [3] computation shows that $\mathrm{K}(G)=\gamma_{2}(G)$ for such groups $G$. So we can assume that $p \geq 5$. Let $G$ be a representative from $\phi_{40}$, which is presented as

$$
\begin{gathered}
G=\left\langle\alpha_{1}, \alpha_{2}, \beta, \beta_{1}, \beta_{2}, \gamma\right|\left[\alpha_{1}, \alpha_{2}\right]=\beta,\left[\beta, \alpha_{i}\right]=\beta_{i},\left[\beta_{1}, \alpha_{2}\right]=\left[\beta_{2}, \alpha_{1}\right]=\gamma, \\
\left.\alpha^{p}=\alpha_{i}{ }^{p}=\beta^{p}=\beta_{i}{ }^{p}=\gamma^{p}=1(i=1,2)\right\rangle .
\end{gathered}
$$

Notice that $\gamma_{2}(G)=\left\langle\beta, \beta_{1}, \beta_{2}, \gamma\right\rangle$ and $\gamma_{4}(G)=\langle\gamma\rangle$. Let $i, j, k \in \mathbb{F}_{p}$. If $j \neq 0$, then

$$
\gamma^{l}=\left[\alpha_{2}{ }^{k j^{-1}} \alpha_{1} \beta^{\frac{1-i}{2}}, \beta_{2}^{-l}\right]
$$

and modulo $\gamma_{4}(G)$ we have

$$
\beta^{i} \beta_{1}^{j} \beta_{2}^{k}=\left[\alpha_{2}^{k j^{-1}} \alpha_{1} \beta^{\frac{1-i}{2}}, \alpha_{2}^{i} \beta^{-j}\right] .
$$

If $j=0$, then

$$
\gamma^{l}=\left[\alpha_{2} \beta^{-\frac{1+i}{2}}, \beta_{1}^{-l}\right]
$$

and modulo $\gamma_{4}(G)$ we have

$$
\beta^{i} \beta_{2}^{k}=\left[\alpha_{2} \beta^{-\frac{1+i}{2}}, \alpha_{1}^{-i} \beta^{-k}\right] .
$$

Thus, for $i, j, k \in \mathbb{F}_{p}$, it follows that

$$
\gamma_{2}(G) / \gamma_{4}(G)=\left(\bigcup_{\substack{i, k \\ j \neq 0}}\left[\bar{\alpha}_{2}^{k j^{-1}} \bar{\alpha}_{1} \bar{\beta}^{\frac{1-i}{2}}, \bar{G}\right]\right) \bigcup\left(\bigcup_{i}\left[\bar{\alpha}_{2} \bar{\beta}^{-\frac{1+i}{2}}, \bar{G}\right]\right),
$$

where $\bar{\alpha}_{1}=\alpha_{1} \gamma_{4}(G), \bar{\alpha}_{2}=\alpha_{2} \gamma_{4}(G)$ and $\bar{\beta}=\beta \gamma_{4}(G)$. Also

$$
\gamma_{4}(G) \subseteq\left(\bigcap_{\substack{i, k \\ j \neq 0}}\left[\alpha_{2}{ }^{k j^{-1}} \alpha_{1} \beta^{\frac{1-i}{2}}, G\right]\right) \bigcap\left(\bigcap_{i}\left[\alpha_{2} \beta^{-\frac{1+i}{2}}, G\right]\right) .
$$

Hence $\mathrm{K}(G)=\gamma_{2}(G)$ by Lemma 2.5 . 
The group $G$, as presented below, is a representative from the isoclinism family $\phi_{41}$ for $p \geq 5$.

$$
\begin{gathered}
G=\left\langle\alpha_{1}, \alpha_{2}, \beta, \beta_{1}, \beta_{2}, \gamma\right|\left[\alpha_{1}, \alpha_{2}\right]=\beta,\left[\beta, \alpha_{i}\right]=\beta_{i},\left[\alpha_{1}, \beta_{1}\right]=\alpha_{1}{ }^{p}=\gamma, \\
\left.\left[\alpha_{2}, \beta_{2}\right]=\gamma^{-\nu}, \alpha^{p}=\alpha_{i}{ }^{p}=\beta^{p}=\beta_{i}{ }^{p}=\gamma^{p}=1(i=1,2)\right\rangle,
\end{gathered}
$$

where $\nu$ denotes the smallest positive integer which is a non-quadratic residue $(\bmod p)$. As in the preceding case, it is easy to check that $\gamma_{2}(G) / \gamma_{4}(G)$ can be written as the union of the sets $\left[\bar{x}, G / \gamma_{4}(G)\right]$, where $\bar{x}$ runs over the elements of the set $S:=\left\{\bar{\alpha}_{2}^{k j^{-1}} \bar{\alpha}_{1} \bar{\beta}^{\frac{1-i}{2}}, \bar{\alpha}_{2} \bar{\beta}^{-\frac{1+i}{2}} \mid i, j(\neq\right.$ $\left.0), k \in \mathbb{F}_{p}\right\}$ and $\gamma_{4}(G)$ is contained in the intersection of the sets $[x, G]$, where $\bar{x} \in S$. Hence $\mathrm{K}(G)=\gamma_{2}(G)$ by invoking Lemma 2.5 again. The proof is now complete by taking $G / H$, where $H$ is any subgroup of $\mathrm{Z}(G)$ of order $p$, and using Theorem 2.9 and Lemma 2.7

Now we take up groups of order $p^{7}$.

Lemma 4.2. Let $L$ be a group of order $p^{7}$ and nilpotency class 4 with $b(L)=3, \mathrm{Z}(L) \leq \gamma_{2}(L)$ and $\gamma_{2}(L)$ elementary abelian of order $p^{4}$. Then $\mathrm{K}(L)=\gamma_{2}(L)$.

Proof. It follows from Lemma 2.4 that $|\mathrm{Z}(L)| \leq p^{2}$. By Theorem 2.11, $L$ admits a subgroup $G$ of order $p^{6}$ and nilpotency class 4 such that $\gamma_{2}(G)=\gamma_{2}(L)$. If $|\mathrm{Z}(G)|=p$, then by Lemma 4.1 we have $\mathrm{K}(G)=\gamma_{2}(G)=\gamma_{2}(L)$. Hence $\mathrm{K}(L)=\gamma_{2}(L)$. So assume that $|\mathrm{Z}(G)|=p^{2}$. By Theorem 2.10, there exists a normal subgroup $H$ of $L$ such that $|L / H: \mathrm{Z}(L / H)|=p^{3}$. We can take $L=\langle a, b, c\rangle$ such that $G=\langle a, b\rangle$. As observed in the proof of Theorem 2.11 (last para), it follows that $H=\gamma_{4}(L)$. Thus $\bar{L}:=L / H=\langle\bar{a}, \bar{b}, \bar{c}\rangle$ is of nilpotency class 3 such that $\bar{c} \in \mathrm{Z}(\bar{L})$, where $\bar{x}=x H$ for any $x \in L$. Since $c \notin \mathrm{Z}(L)$, we have $[c, L]=H$. Notice that $\gamma_{2}(\bar{L})=\langle[\bar{a}, \bar{b}],[\bar{a},[\bar{a}, \bar{b}]],[\bar{b},[\bar{a}, \bar{b}]]\rangle$. Let $\alpha_{1}, \alpha_{2}, \alpha_{3} \in \mathbb{F}_{p}$. If $\alpha_{3} \neq 0$, then we can write

$$
[\bar{a}, \bar{b}]^{\alpha_{1}}[\bar{b},[\bar{a}, \bar{b}]]^{\alpha_{2}}[\bar{a},[\bar{a}, \bar{b}]]^{\alpha_{3}}=\left[\bar{a} \bar{b}^{\alpha_{2} \alpha_{3}{ }^{-1}}[\bar{a}, \bar{b}]^{\frac{1-\alpha_{1}}{2}}, \bar{b}^{\alpha_{1}}[\bar{a}, \bar{b}]^{\alpha_{3}}\right] .
$$

If $\alpha_{3}=0$, then we can write

$$
[\bar{a}, \bar{b}]^{\alpha_{1}}[\bar{b},[\bar{a}, \bar{b}]]^{\alpha_{2}}=\left[\bar{b}[\bar{a}, \bar{b}]^{-\frac{1+\alpha_{1}}{2}}, \bar{a}^{-\alpha_{1}}[\bar{a}, \bar{b}]^{\alpha_{2}}\right] .
$$

Hence, for $i, j \in \mathbb{F}_{p}$ and $\epsilon=0,1$ such that $i$ and $\epsilon$ are not simultaneously zero, we have

$$
\gamma_{2}(\bar{L})=\bigcup_{\epsilon, i, j}\left[\bar{a}^{\epsilon} \bar{b}^{i}[\bar{a}, \bar{b}]^{j}, \bar{L}\right]
$$

Notice that $G$ lies in the isoclinism family $\phi_{23}$ of [14]. Therefore we can take $H=\left\langle\left[b, \gamma_{3}(G)\right]\right\rangle$. Since $\mathrm{C}_{G}(c)$ is maximal, by suitably modifing $c$, we can assume that $[b, c]=1$ and $H=\langle[a, c]\rangle$. Then, for all $i, j \in \mathbb{F}_{p}$ and $\epsilon=0,1$, it follows that $H \subseteq\left[a^{\epsilon} b^{i}[\bar{a}, \bar{b}]^{j}, L\right]$, where $i$ and $\epsilon$ are not simultaneously zero. Hence $\mathrm{K}(L)=\gamma_{2}(L)$ by Lemma 2.5 .

\section{Groups of CLASS 3 AND ORDER $p^{7}$}

In this section we take up groups $G$ of order $p^{7}, p$ odd, and nilpotency class 3 , and prove that $\mathrm{K}(G)=\gamma_{2}(G)$ if $|\mathrm{Z}| \leq p^{2}$ and $\mathrm{K}(G) \neq \gamma_{2}(G)$ otherwise. A group $G$ is said to be a freest group of nilpotency class 2 on $n$ generators if $G=F_{n} / \gamma_{3}\left(F_{n}\right)$ and $|G|=p^{n(n+1) / 2}$, where $F_{n}$ denotes the free group on $n$ generators. Throughout the remaining of the paper $\epsilon \in\{0,1\}$. 
Lemma 5.1. Let $G$ be a group of order $p^{7}$ and nilpotency class 3 with $b(G)=3,|\mathrm{Z}(G)| \leq p^{2}$ and $\gamma_{2}(G)$ elementary abelian of order $p^{4}$. Then $\mathrm{K}(G)=\gamma_{2}(G)$.

Proof. Notice that $G$ is a stem group in its isoclinism class. In view of Lemma 2.3, it follows that $G$ is minimally generated by 3 elements $a, b, c$ (say). For notational convenience, set $\mathrm{C}=\mathrm{C}_{G}\left(\gamma_{2}(G)\right)$. To enhance the readability of the proof, we divide it in several steps.

Step 1. If $|\mathrm{Z}(G)|=p$, then $\mathrm{C}=\gamma_{2}(G)$.

Proof. Since $|Z(G)|=p$, we have $\left|\gamma_{3}(G)\right|=p$, and therefore no non trivial element from the subgroup $\langle[a, b],[a, c],[b, c]\rangle$ can lie in $\gamma_{3}(G)$. If $|\mathrm{C}|=p^{6}$, then, without loss of generality, we can assume that $b, c \in \mathrm{C}$. By Hall-Witt Identity we have

$$
[[a, b], c][[c, a], b][[b, c], a]=1,
$$

which implies that $[b, c] \in \mathrm{Z}(G)$. As observed above, this is not possible.

If $|\mathrm{C}|=p^{5}$, then, without loss of generality, we can assume that $c \in \mathrm{C}$. If both $[a,[a, b]]$ and $[b,[a, b]]$ are trivial, then $[a, b] \in Z(G)$, which is not possible. By symmetry, we can assume that $\gamma_{3}(G)=\langle[a,[a, b]]\rangle$. Notice, by Hall-Witt Identity, that $[a,[b, c]]=[b,[a, c]]$. First we assume that $[a,[b, c]]$ is trivial. If $[b,[b, c]]$ or $[a,[a, c]]$ is trivial, then $[b, c]$ or $[a, c]$, respectively, lies in $Z(G)$, which is again not possible. So assume that both $[b,[b, c]]$ and $[a,[a, c]]$ are non trivial. Then $[b,[a, b]]=[b,[b, c]]^{t}$ for some $t \in \mathbb{F}_{p}$. Also $[a,[a, b]]=[a,[a, c]]^{s}$ for some $s \in \mathbb{F}_{p}^{*}$. Hence both $\left[b,[a, b][b, c]^{-t}\right]$ and $\left[a,[a, b][a, c]^{-s}\right]$ are trivial, which implies that $[a, b][a, c]^{-s}[b, c]^{-t} \in Z(G)$, not possible.

Finally assume that $[a,[b, c]]$ is non trivial. Then $[b,[a, c]]$ is also non trivial. If $[b,[b, c]]$ is non trivial, then $[a,[b, c]]=[b,[b, c]]^{s}$ for some $s \in \mathbb{F}_{p}^{*}$, and therefore $\left[a b^{-s},[b, c]\right]=1$. By replacing $a$ by $a b^{-s}$, we get a modified generating set $\{a, b, c\}$ for $G$ such that $c \in \mathrm{C}$ and $[a,[b, c]]=1$. Similarly, if $[a,[a, c]]$ is non trivial, then we can modify the generating set for $G$ such that $[b,[a, c]]=1$. So, in both the cases, we land up in first case, which we have already handled. So let $[b,[b, c]]=[a,[a, c]]=1$. Then $[a,[a, b]][a,[b, c]]^{-s}=1$, which implies that $\left[a,[a, b][b, c]^{-s}\right]=1$ for some $s \in \mathbb{F}_{p}^{*}$. Similarly $\left[b,[a, b][a, c]^{-t}\right]=1$ for some $t \in \mathbb{F}_{p}$. This implies that $[a, b][b, c]^{-s}[a, c]^{-t} \in \mathrm{Z}(G)$, which is not possible. Thus $\mathrm{C}$ can not have order $p^{5}$. Hence $\mathrm{C}=\gamma_{2}(G)$.

Step 2. If $|\mathrm{Z}(G)|=p$, then $\mathrm{K}(G)=\gamma_{2}(G)$.

Proof. By Step 1 we have $\mathrm{C}=\gamma_{2}(G)$. Notice that $\bar{G}:=G / \gamma_{3}(G)$ is the 3 -generated freest group of nilpotency class 2 and order $p^{6}$. Let $\alpha_{i} \in \mathbb{F}_{p}, 1 \leq i \leq 3$. For $\alpha_{1} \neq 0$, we have

$$
[\bar{a}, \bar{b}]^{\alpha_{1}}[\bar{b}, \bar{c}]^{\alpha_{2}}[\bar{a}, \bar{c}]^{\alpha_{3}}=\left[\bar{a} \bar{c}^{-\alpha_{2} \alpha_{1}{ }^{-1}}, \bar{b}^{\alpha_{1}} \bar{c}^{\alpha_{3}}\right],
$$

where $\bar{x}=x \gamma_{3}(G)$ for $x \in G$. Also, for $\alpha_{1}=0$, we have

$$
[\bar{b}, \bar{c}]^{\alpha_{2}}[\bar{a}, \bar{c}]^{\alpha_{3}}=\left[\bar{c}, \bar{a}^{-\alpha_{3}} \bar{b}^{-\alpha_{2}}\right] .
$$

Hence for $i \in \mathbb{F}_{p}$, we get

$$
\gamma_{2}(\bar{G})=\bigcup_{\epsilon, i}\left[\bar{a}^{\epsilon} \bar{c}^{i}, \bar{G}\right]
$$

Since $\bar{G}$ is the freest group, by symmetry we can interchange $a, b, c$ in the preceding equation. 
To complete the proof of this step, it is sufficient to show that

$$
\gamma_{3}(G) \subseteq \bigcap_{\epsilon, i}\left[x^{\epsilon} y^{i}, G\right]
$$

for some $x \neq y$ in $\{a, b, c\}$, where $i \in \mathbb{F}_{p}$ such that $\epsilon$ and $i$ are not simultaneously zero. First assume that $[a,[a, b]] \neq 1$, and therefore generates $\gamma_{3}(G)$. If $[c,[a, b]] \neq 1$, then $[c,[a, b]]=$ $[a,[a, b]]^{t}$ for some $t \in \mathbb{F}_{p}^{*}$. Therefore, by modifying $c$ by $c a^{-t}$, we get a new generating set $\{a, b, c\}$ for $G$ such that $[c,[a, b]]=1$ and $[a,[a, b]] \neq 1$. So we can always assume that $[c,[a, b]]=1$. Since $c \notin \mathrm{C}$, either $[c,[b, c]]$ or $[c,[a, c]]$ is non trivial. Hence for $\epsilon=0,1$ and $i \in \mathbb{F}_{p}$, not simultaneously zero, it is easy to see that $\gamma_{3}(G) \subseteq \bigcap_{\epsilon, i}\left[a^{\epsilon} c^{i}, G\right]$.

Now let us assume that $[a,[a, b]]=1$. Then, notice that, at least one of $[a,[b, c]]$ and $[a,[a, c]]$ is not trivial. If $[b,[a, b]]=1$, then $[c,[a, b]] \neq 1$. Hence for $\epsilon=0,1$ and $i \in \mathbb{F}_{p}$ not simultaneously zero, we get $\gamma_{3}(G) \subseteq \bigcap_{\epsilon, i}\left[c^{\epsilon} a^{i}, G\right]$. If $[b,[a, b]] \neq 1$, then $\gamma_{3}(G) \subseteq \bigcap_{\epsilon, i}\left[b^{\epsilon} a^{i}, G\right]$, where both $\epsilon$ and $i$ are not simultaneously zero.

Step 3. If $|\mathrm{Z}(G)|=p^{2}$ and $\left|\gamma_{3}(G)\right|=p$, then $|\mathrm{C}|=p^{5}$.

Proof. If $|\mathrm{C}|=p^{6}$, then, without loss of generality, we can assume that $b, c \in \mathrm{C}$, and therefore by Hall-Witt Identity it follows that $[b, c] \in \mathrm{Z}(G)$. Hence no non-trivial element of the form $[a, b]^{r}[a, c]^{s}$ can lie in $\mathrm{Z}(G)$, where $r, s \in \mathbb{F}_{p}$. If either $[a,[a, b]]$ or $[a,[a, c]]$ is trivial, then, $\gamma_{3}(G)$ being of order $p$, it follows that either $[a, b]$ or $[a, c]$, respectively, lie in $Z(G)$, which is not possible. If both $[a,[a, b]]$ and $[a,[a, c]]$ are non trivial, then $[a,[a, b]]=[a,[a, c]]^{t}$ for some $t \in \mathbb{F}_{p}^{*}$. Hence $[a, b][a, c]^{-t} \in Z(G)$, which is again not possible. Hence $|\mathrm{C}| \neq p^{6}$.

If $|\mathrm{C}|=p^{4}$, then $\mathrm{C}=\gamma_{2}(G)$. By a suitable modification in the generating set for $G$, we can assume that $[b, c] \in Z(G)$. Indeed, if one of $[a, b],[a, c]$ and $[b, c]$ is in $\mathrm{Z}(G)$, then, after suitably renaming the generators, we can assume that $[b, c] \in \mathrm{Z}(G)$. If not, then, after renaming the generators, if necessary, we can assume that $[b, c]=[a, b]^{r}[a, c]^{s}$ modulo $\mathrm{Z}(G)$ for some $r, s \in \mathbb{F}_{p}$. This implies that, modulo $\mathrm{Z}(G),\left[b a^{-s}, c a^{r}\right]=1$. Thus the new generating set $\left\{a, b a^{-s}, c a^{r}\right\}$ has the required property. Once $[b, c] \in \mathrm{Z}(G)$, notice that both $[a,[a, b]]$ and $[a,[a, c]]$ can not be trivial; otherwise $a$ will lie in $\mathrm{C}$, which can not happen. By symmetry, we can assume that $[a,[a, b]]$ is non trivial and therefore generates $\gamma_{3}(G)$. By Hall-Witt Identity we have $[c,[a, b]]=[b,[a, c]]$. We first assume that $[c,[a, b]]$ is trivial. Then none of the two elements $[c,[a, c]]$ and $[b,[a, b]]$ can be trivial. Indeed, if $[x,[a, x]]=1$, then $x \in \mathrm{C}$ for $x=$ $b, c$, which is not the case. Then $[a,[a, c]]=[c,[a, c]]^{r}$ for some $r \in \mathbb{F}_{p}$, which implies that $\left[a c^{-r},[a, c]\right]=1$. Replacing $a$ by $a c^{-r}$, we get a generating set $\left\{a^{\prime}=a c^{-r}, b, c\right\}$ such that $\gamma_{2}(G)=\left\langle\left[a^{\prime}, b\right],\left[a^{\prime}, c\right],[b, c],\left[a^{\prime},\left[a^{\prime}, b\right]\right]\right\rangle$. A straightforward computation shows that $[b, c] \in Z(G)$ and $\left[b,\left[a^{\prime}, c\right]\right]=\left[c,\left[a^{\prime}, b\right]\right]=1=\left[a^{\prime},\left[a^{\prime}, c\right]\right]$; but $\left[c,\left[a^{\prime}, c\right]\right]$ and $\left[b,\left[a^{\prime}, b\right]\right]$ are non trivial. Now $\left[a^{\prime},\left[a^{\prime}, b\right]\right]=\left[b,\left[a^{\prime}, b\right]\right]^{t}$ for some $t \in \mathbb{F}_{p}^{*}$, which implies that $a^{\prime} b^{-t}$ lies in $\mathrm{C}$, which is not possible.

Now we claim that when at least one the two elements $[b,[a, b]]$ and $[c,[a, c]]$ is non trivial, then we can modify the generating set for $G$ such that $[c,[a, b]]=1$, but $[b,[a, b]]$ and $[c,[a, c]]$ are both non trivial, and hence we fall in the preceding case. If $[b,[a, b]]$ is non trivial, then $[c,[a, b]]=[b,[a, b]]^{t}$ for some $t \in \mathbb{F}_{p}$. Replacing $c$ by $b^{-t} c$, we get the required generating set. If $[c,[a, c]]$ is non trivial, then $[b,[a, c]]=[c,[a, c]]^{r}$ for some $r \in \mathbb{F}_{p}$. Now replacing $b$ by $c^{-r} b$, we again get the required generating set. 
Finally assume that $[b,[a, c]]=[c,[a, b]]$ is non trivial, and $[b,[a, b]]$ and $[c,[a, c]]$ are both trivial. Then $[a,[a, c]]=[b,[a, c]]^{r}$ for some $r \in \mathbb{F}_{p}$, which gives $\left[a b^{-r},[a, c]\right]=1$. Replacing $a$ by $a b^{-r}$, we get a generating set $\left\{a^{\prime}=a b^{-r}, b, c\right\}$ for $G$ such that $\gamma_{2}(G)=\left\langle\left[a^{\prime}, b\right],\left[a^{\prime}, c\right],[b, c],\left[a^{\prime},\left[a^{\prime}, b\right]\right]\right\rangle$, with $[b, c] \in Z(G)$ and $\left[a^{\prime},\left[a^{\prime}, c\right]\right]=1$. Notice that $\left[a^{\prime},\left[a^{\prime}, b\right]\right]=[a,[a, b]]$ and $\left[c,\left[a^{\prime}, b\right]\right]=[c,[a, b]]$, both of which are non trivial. Hence $\left[a^{\prime},\left[a^{\prime}, b\right]\right]=\left[c,\left[a^{\prime}, b\right]\right]^{t}$ for some $t \in \mathbb{F}_{p}^{*}$, which gives $\left[a^{\prime} c^{-t},\left[a^{\prime}, b\right]\right]=1$. Since $\left[c,\left[a^{\prime}, c\right]\right]=[c,[a, c]]=1$, it follows that $\left[a^{\prime} c^{-t},\left[a^{\prime}, c\right]\right]=1$. Hence $a^{\prime} c^{-t} \in \mathrm{C}$, which is not possible. We have handled all the cases, and the proof of this step is complete.

Step 4. If $|\mathrm{Z}(G)|=p^{2}$ and $\left|\gamma_{3}(G)\right|=p$, then $\mathrm{K}(G)=\gamma_{2}(G)$.

Proof. By Step 3, we know that $|\mathrm{C}|=p^{5}$. Without loss of generality we can assume that $c \in C$. We consider three different cases, namely $[b, c] \in \mathrm{Z}(G),[a, c] \in \mathrm{Z}(G)$ and otherwise. Since $\bar{G}:=G / \gamma_{3}(G)$ is the freest group, then, as explained in Step 2, for proving $\mathrm{K}(G)=\gamma_{2}(G)$, it is sufficient to show that

$$
\gamma_{3}(G) \subseteq \bigcap_{\epsilon, i}\left[x^{\epsilon} y^{i}, G\right]
$$

for some $x \neq y$ in $\{a, b, c\}$, where $\epsilon=0,1$ and $i \in \mathbb{F}_{p}$ such that $\epsilon$ and $i$ are not simultaneously zero.

Case (i). Let $[b, c] \in \mathrm{Z}(G)$. Notice that all $[a,[b, c]],[c,[a, b]]$ and $[b,[a, c]]$ are trivial. Then none of the two elements $[a,[a, c]]$ and $[b,[a, b]]$ can be trivial. Thus for any $t, i \in \mathbb{F}_{p}$ we have $[a,[a, c]]^{t}=\left[a b^{i},[a, c]^{t}\right]$ and $[b,[a, b]]^{t}=\left[b,[a, b]^{t}\right]$, which implies that

$$
\gamma_{3}(G)=\bigcap_{\epsilon, i}\left[a^{\epsilon} b^{i}, \gamma_{2}(G)\right] \subseteq \bigcap_{\epsilon, i}\left[a^{\epsilon} b^{i}, G\right],
$$

where $\epsilon$ and $i$ are not simultaneously zero. Hence $\mathrm{K}(G)=\gamma_{2}(G)$ by Lemma 2.5 .

Case (ii). Next assume that $[a, c] \in \mathrm{Z}(G)$. As in the preceding case, both the elements $[a,[a, b]]$ and $[b,[b, c]]$ are non trivial and $[a,[b, c]]=1$. For any $t, i \in \mathbb{F}_{p}$ we have $[b,[b, c]]^{t}=\left[b a^{i},[b, c]^{t}\right]$ and $[a,[a, b]]^{t}=\left[a,[a, b]^{t}\right]$. Thus for $\epsilon=0,1$ and $i \in \mathbb{F}_{p}$, not simultaneously zero, we get

$$
\gamma_{3}(G)=\bigcap_{\epsilon, i}\left[b^{\epsilon} a^{i}, \gamma_{2}(G)\right] \subseteq \bigcap_{\epsilon, i}\left[b^{\epsilon} a^{i}, G\right]
$$

Case (iii). Let neither $[b, c]$ nor $[a, c]$ lie in $\mathrm{Z}(G)$. We can assume that none of the two elements $[b, c]$ and $[a, c]$ can be a power of the other. For, if $[b, c]=[a, c]^{t}$, for some $t \in \mathbb{F}_{p}^{*}$, then we can take a generating set $\left\{a, b a^{-t}, c\right\}$ such that $\left[b a^{-t}, c\right] \in \mathrm{Z}(G)$, and we fall in Case (i). We now modify the generating set for $G$ to $\left\{a^{\prime}, b^{\prime}, c\right\}$ such that $\left[a^{\prime}, b^{\prime}\right] \in \mathrm{Z}(G)$ and $c \in \mathrm{C}$. If $[a, b] \in \mathrm{Z}(G)$, then obviously we take $\left\{a^{\prime}=a, b^{\prime}=b, c\right\}$. If not, then, modulo $\mathrm{Z}(G),[a, b]=$ $[a, c]^{r}[b, c]^{s}$ for some $r, s \in \mathbb{F}_{p}$. This implies that, modulo $\mathrm{Z}(G),\left[a c^{s}, b c^{-r}\right]=1$. Thus if we take $\left\{a^{\prime}=a c^{s}, b^{\prime}=b c^{-r}, c\right\}$ as a generating set for $G$, then $\left[a^{\prime}, b^{\prime}\right] \in \mathrm{Z}(G)$ and $c$ still lies in C.

First assume that $\left[a^{\prime},\left[a^{\prime}, c\right]\right]=\left[b^{\prime},\left[b^{\prime}, c\right]\right]=1$. Since $a^{\prime}, b^{\prime} \notin C$, we have $\left[a^{\prime},\left[b^{\prime}, c\right]\right]=\left[b^{\prime},\left[a^{\prime}, c\right]\right] \neq$ 1. For any $t, i \in \mathbb{F}_{p}$ we have $\left[a^{\prime},\left[b^{\prime}, c\right]\right]^{t}=\left[a^{\prime} b^{\prime i},\left[b^{\prime}, c\right]^{t}\right]$ and $\left[b^{\prime},\left[a^{\prime}, c\right]\right]^{t}=\left[b^{\prime},\left[a^{\prime}, c\right]^{t}\right]$. Thus for $\epsilon=0,1$ and $i \in \mathbb{F}_{p}$, not simultaneously zero, we get

$$
\gamma_{3}(G)=\bigcap_{\epsilon, i}\left[a^{\epsilon} b^{i}, \gamma_{2}(G)\right] \subseteq \bigcap_{\epsilon, i}\left[a^{\epsilon} b^{i}, G\right] .
$$

Now assume that $\left[b^{\prime},\left[b^{\prime}, c\right]\right]$ or $\left[a^{\prime},\left[a^{\prime}, c\right]\right]$ is non trivial. We only present the proof when $\left[b^{\prime},\left[b^{\prime}, c\right]\right] \neq 1$. The other case goes on similar lines. Without loss of generality, we can assume 
$\left[a^{\prime},\left[b^{\prime}, c\right]\right]=\left[b^{\prime},\left[a^{\prime}, c\right]\right]=1$. Indeed, if $\left[b^{\prime},\left[a^{\prime}, c\right]\right]=1$, then nothing to be done. Otherwise $\left[a^{\prime},\left[b^{\prime}, c\right]\right]=\left[b^{\prime},\left[b^{\prime}, c\right]\right]^{s}$, which gives $\left[a^{\prime} b^{\prime-s},\left[b^{\prime}, c\right]\right]=1$. Replacing $a^{\prime}$ by $a^{\prime} b^{\prime-s}$, we get the required generating set $\left\{\tilde{a}=a^{\prime} b^{\prime^{-s}}, b^{\prime}, c\right\}$ for which $\left[b^{\prime},[\tilde{a}, c]\right]=\left[\tilde{a},\left[b^{\prime}, c\right]\right]=1,\left[\tilde{a}, b^{\prime}\right] \in Z(G), c \in C$ and $[\tilde{a},[\tilde{a}, c]] \neq 1$. For any $t, i \in \mathbb{F}_{p}$ we have $[\tilde{a},[\tilde{a}, c]]^{t}=\left[\tilde{a} b^{i},[\tilde{a}, c]^{t}\right]$ and $\left[b^{\prime},\left[b^{\prime}, c\right]\right]^{t}=\left[b^{\prime},\left[b^{\prime}, c\right]^{t}\right]$. Thus for $\epsilon=0,1$ and $i \in \mathbb{F}_{p}$, not simultaneously zero, we get

$$
\gamma_{3}(G)=\bigcap_{\epsilon, i}\left[a^{\epsilon} b^{i}, \gamma_{2}(G)\right] \subseteq \bigcap_{\epsilon, i}\left[a^{\epsilon} b^{i}, G\right]
$$

Hence $\mathrm{K}(G)=\gamma_{2}(G)$, which completes the proof of Step 4 .

It only remains to handle the situation when $|\mathrm{Z}(G)|=\left|\gamma_{3}(G)\right|=p^{2}$. We claim that $b(G)=4$ in this case. Contrarily assume that $b(G)=3$. Then by Theorem 2.10 there exists a normal subgroup $H$ of $G$ such that $|G / H: \mathrm{Z}(G / H)|=p^{3}$. Since $\mathrm{Z}(G)=\gamma_{3}(G)$ is of order $p^{2}$, the nilpotency class of $G / H$ is 3 . So we can assume that $c H \in \mathrm{Z}(G / H)$. Notice that $\left|\gamma_{2}(G / H) / \gamma_{3}(G / H)\right|$ must be $p^{2}$, which is not possible as $G / H$ admits only two non-central generators. Hence the claim follows, and the proof of the lemma is complete

We now prove

Lemma 5.2. Let $G$ be a group of order $p^{7}$ and nilpotency class 3 with $b(G)=3,|Z(G)|=p^{3}$ and $\gamma_{2}(G)$ elementary abelian of order $p^{4}$, where $p$ is an odd prime. Then $\mathrm{K}(G) \neq \gamma_{2}(G)$. Moreover, every element of $\gamma_{2}(G)$ can be written as a product of at most two elements from $\mathrm{K}(G)$.

Proof. We start by noticing that $\left|\gamma_{3}(G)\right| \leq p^{2}$. Let $G=\langle a, b, c\rangle$. Then $\gamma_{2}(G)=\langle[a, b],[b, c],[a, c]$, $\left.\gamma_{3}(G)\right\rangle$. Without loss of generality we can assume that $Z(G)=\left\langle[b, c],[a, c], \gamma_{3}(G)\right\rangle$. Indeed, modulo $\mathrm{Z}(G)$, we can assume that $c$ commutes with $a$ and $b$. This, by Hall-Witt Identity, implies that $c \in \mathrm{C}_{G}\left(\gamma_{2}(G)\right)$. Now we consider two cases, namely, $\left|\gamma_{3}(G)\right|$ is $p$ or $p^{2}$.

First assume $\left|\gamma_{3}(G)\right|=p$. By suitably modifying the generating set $\{a, b, c\}$, if necessary, we can assume that $[a,[a, b]] \neq 1$ and $[b,[a, b]]=1$. Hence $\gamma_{3}(G)=\langle[a,[a, b]]\rangle$. Now we claim that $[b, c][a,[a, b]]$ is not a commutator. Contrarily assume that

$$
[b, c][a,[a, b]]=\left[a^{\alpha_{1}} b^{\alpha_{2}} c^{\alpha_{3}}[a, b]^{\alpha_{4}}, a^{\beta_{1}} b^{\beta_{2}} c^{\beta_{3}}[a, b]^{\beta_{4}}\right],
$$

where $\alpha_{i}, \beta_{i} \in \mathbb{F}_{p}$. After solving and comparing the powers, we get

$$
\begin{aligned}
\beta_{2} \alpha_{1}-\alpha_{2} \beta_{1} & =0, \\
\beta_{3} \alpha_{2}-\alpha_{3} \beta_{2} & =1, \\
\beta_{3} \alpha_{1}-\alpha_{3} \beta_{1} & =0, \\
\alpha_{2}\left(\begin{array}{c}
\beta_{1} \\
2
\end{array}\right)-\beta_{2}\left(\begin{array}{c}
\alpha_{1} \\
2
\end{array}\right)+\beta_{4} \alpha_{1}-\alpha_{4} \beta_{1} & =1 .
\end{aligned}
$$

If $\alpha_{2}=0$, then from (5.2), we get $\alpha_{3}, \beta_{2}$ are non zero. So (5.1) gives $\alpha_{1}=0$, which in turn, using (5.3) gives $\beta_{1}=0$. But these values contradict (5.4). Now let $\alpha_{2} \neq 0$. Then from (5.1), $\beta_{1}=\beta_{2} \alpha_{1} \alpha_{2}{ }^{-1}$. Substituting $\beta_{1}$ in (5.4), we get

$$
\alpha_{1}\left(\beta_{4}+\alpha_{2}^{-1} \beta_{2}\left(\left(\alpha_{1} \beta_{2}-\alpha_{2} \beta_{2}\right) / 2-\alpha_{4}\right)\right)=1 ;
$$

hence $\alpha_{1} \neq 0$. Substituting $\beta_{1}$ in (5.3) , we get $\alpha_{1}\left(\beta_{3}-\alpha_{3} \beta_{2} \alpha_{2}{ }^{-1}\right)=0$. Hence $\beta_{3}=\alpha_{3} \beta_{2} \alpha_{2}{ }^{-1}$, which contradicts (5.2). Our claim is now settled. 
Now we assume $\left|\gamma_{3}(G)\right|=p^{2}$. Thus $\gamma_{3}(G)=\langle[a,[a, b]],[b,[a, b]]\rangle$. Notice that if neither $[a, c]$ nor $[b, c]$ lies in $\gamma_{3}(G)$, then one of them will be a power of the other modulo $\gamma_{3}(G)$. Then we can modify the generating set $\{a, b, c\}$ such that $[a, c] \in \gamma_{3}(G)$, without disturbing other setup. Let $[a, c]=[a,[a, b]]^{t_{1}}[b,[a, b]]^{t_{2}}$ for some $t_{1}, t_{2} \in \mathbb{F}_{p}$. Then $\left[a, c[a, b]^{-t_{1}}\right]=[b,[a, b]]^{t_{2}}$. By replacing $c$ with $c[a, b]^{-t_{1}}$, we get a modified generating set for $G$, which we still call $\{a, b, c\}$, such that $[a, c]=[b,[a, b]]^{t_{2}}$ and $c \in \mathrm{C}_{G}\left(\gamma_{2}(G)\right)$. If $t_{2}=0$, then $[a, c]=1$, otherwise we can replace $c$ by $c^{t_{2}^{-1}}$, and assume that $t_{2}=1$; hence $[a, c]=[b,[a, b]]$.

Let us assume that for given $\lambda, \nu \in \mathbb{F}_{p}^{*}$, there exist $\alpha_{i}, \beta_{i} \in \mathbb{F}_{p}$ such that

$$
[b, c]^{\lambda}[a,[a, b]]^{\nu}=\left[a^{\alpha_{1}} b^{\alpha_{2}} c^{\alpha_{3}}[a, b]^{\alpha_{4}}, a^{\beta_{1}} b^{\beta_{2}} c^{\beta_{3}}[a, b]^{\beta_{4}}\right] .
$$

After solving and comparing the powers on both sides, we get

$$
\begin{aligned}
\beta_{2} \alpha_{1}-\alpha_{2} \beta_{1} & =0 \\
\beta_{3} \alpha_{2}-\alpha_{3} \beta_{2} & =\lambda \\
\beta_{1} \beta_{2} \alpha_{2}-\alpha_{1} \alpha_{2} \beta_{2}-\alpha_{1}\left(\begin{array}{c}
\beta_{2} \\
2
\end{array}\right)+\beta_{1}\left(\begin{array}{c}
\alpha_{2} \\
2
\end{array}\right)+\left(\beta_{3} \alpha_{1}-\alpha_{3} \beta_{1}\right)+\beta_{4} \alpha_{2}-\alpha_{4} \beta_{2} & =0 \\
\alpha_{2}\left(\begin{array}{c}
\beta_{1} \\
2
\end{array}\right)-\beta_{2}\left(\begin{array}{c}
\alpha_{1} \\
2
\end{array}\right)+\beta_{4} \alpha_{1}-\alpha_{4} \beta_{1} & =\nu .
\end{aligned}
$$

Using (5.5) in (5.7) and (5.8), we, respectively, get

$$
\begin{aligned}
\left(\beta_{1} \beta_{2} \alpha_{2}-\alpha_{1} \alpha_{2} \beta_{2}\right) / 2+\left(\beta_{3} \alpha_{1}-\alpha_{3} \beta_{1}\right)+\beta_{4} \alpha_{2}-\alpha_{4} \beta_{2} & =0 \\
\left(\beta_{1} \beta_{2} \alpha_{1}-\alpha_{1} \alpha_{2} \beta_{1}\right) / 2+\beta_{4} \alpha_{1}-\alpha_{4} \beta_{1} & =\nu
\end{aligned}
$$

We proceed in two different cases, namely, $[a, c]=1$ and $[a, c]=[b,[a, b]]$.

Case(1). Let $[a, c]=1$. Then (5.9) reduces to

$$
\left(\beta_{1} \beta_{2} \alpha_{2}-\alpha_{1} \alpha_{2} \beta_{2}\right) / 2+\beta_{4} \alpha_{2}-\alpha_{4} \beta_{2}=0 .
$$

If $\alpha_{2}=0$, then by (5.6), we get $\beta_{2} \neq 0$, which using, (5.5), gives $\alpha_{1}=0$. Hence by (5.11), $\alpha_{4}=0$, which contradicts (5.10). If $\alpha_{2} \neq 0$ and $\alpha_{1}=0$, then by (5.5) $\beta_{1}=0$, which contradicts (5.10). Hence, $\alpha_{2} \neq 0$ implies $\alpha_{1} \neq 0$. By symmetry we can take both $\beta_{1}$ and $\beta_{2}$ non zero. Hence we can assume that $\alpha_{1}, \alpha_{2}, \beta_{1}, \beta_{2}$ are all nonzero.

Computing the value of $\alpha_{4}$ from (5.11) and substituting in (5.10), we get

$$
\left(\beta_{1} \beta_{2} \alpha_{1}-\alpha_{1} \alpha_{2} \beta_{1}\right) / 2+\beta_{4} \alpha_{1}-\left(\left(\beta_{1} \alpha_{2}-\alpha_{1} \alpha_{2}\right) / 2+\beta_{4} \alpha_{2} \beta_{2}{ }^{-1}\right) \beta_{1}=\nu .
$$

Using (5.5), it is easy to see that the left hand side of the preceding equation is zero, which contradicts the choice of $\nu$. Hence for any $\lambda, \nu \in \mathbb{F}_{p}^{*},[b, c]^{\lambda}[a,[a, b]]^{\nu}$ is not a commutator.

Case(2). Let $[a, c]=[b,[a, b]]$. If $\alpha_{2}=0$, then by (5.5), $\alpha_{1}=0$, and the equations (5.6), (5.9) and (5.10), respectively, reduces to

$$
\begin{aligned}
\alpha_{3} \beta_{2} & =-\lambda \\
\alpha_{3} \beta_{1}+\alpha_{4} \beta_{2} & =0 \\
\alpha_{4} \beta_{1} & =-\nu .
\end{aligned}
$$

Solving this we get $\left(\beta_{1}{ }^{-1} \beta_{2}\right)^{2}=-\lambda \nu^{-1}$, which is not possible for a choice of $\lambda$ and $\mu$ such that $-\lambda \nu^{-1}$ is a non-quadratic residue mod $p$. Hence for any such $\lambda, \nu \in \mathbb{F}_{p}^{*},[b, c]^{\lambda}[a,[a, b]]^{\nu}$ is not a commutator. If $\alpha_{2} \neq 0$ and $\alpha_{1}=0$, then by (5.5) $\beta_{1}=0$, which contradicts (5.10). Hence, 
$\alpha_{2} \neq 0$ implies $\alpha_{1} \neq 0$. By symmetry we can take both $\beta_{1}$ and $\beta_{2}$ non zero. Hence we can assume that $\alpha_{1}, \alpha_{2}, \beta_{1}, \beta_{2}$ are all nonzero.

Using (5.5) in (5.9) and (5.10), we, respectively, get

$$
\alpha_{4}=\left(\beta_{1} \alpha_{2}-\alpha_{1} \alpha_{2}\right) / 2+\beta_{2}^{-1}\left(\beta_{3} \alpha_{1}-\alpha_{3} \beta_{1}\right)+\beta_{4} \alpha_{2} \beta_{2}{ }^{-1}
$$

and

$$
\alpha_{4}=\left(\beta_{2} \alpha_{1}-\alpha_{1} \alpha_{2}\right) / 2+\beta_{4} \alpha_{1} \beta_{1}{ }^{-1}-\nu \beta_{1}{ }^{-1} .
$$

Equating these equations and using (5.5), we get $\left(\beta_{3} \alpha_{1}-\alpha_{3} \beta_{1}\right)=-\nu \beta_{1}{ }^{-1} \beta_{2}$. Multiply both sides by $\beta_{1}^{-1} \beta_{2}$ and using (5.5) and (5.6), we further get $\left(\beta_{1}{ }^{-1} \beta_{2}\right)^{2}=-\lambda \nu^{-1}$. Hence, as above, for $\lambda$ and $\nu$ such that $-\lambda \nu^{-1}$ is a non-quadratic residue $\bmod p,[b, c]^{\lambda}[a,[a, b]]^{\nu}$ is not a commutator. The proof is now complete by taking $G / H$, where $H$ is any subgroup of $\mathrm{Z}(G)$ of order $p$, and using Theorem 2.9 and Lemma 2.7

\section{Proof of Theorem A}

In this section we present a proof of Theorem A. The following result is the last thread to accomplish our endeavour.

Lemma 6.1. Let $L$ be a finite $p$-group of order at least $p^{8}$ and nilpotency class 3 with $b(L)=3$, $|\mathrm{Z}(L)|=p^{3}, \mathrm{Z}(L) \leq \gamma_{2}(L)$ and $\gamma_{2}(L)$ elementary abelian of order $p^{4}$. Then $\mathrm{K}(L)=\gamma_{2}(L)$.

Proof. Notice that $\left|\gamma_{3}(L)\right| \leq p^{2}$. We first assume that $\left|\gamma_{3}(L)\right|=p$. Then by Theorem 2.10 there exists a normal subgroup $H$ of $L$ such that $|L / H: \mathrm{Z}(L / H)|=p^{3}$. If $H \neq \gamma_{3}(L)$, then it follows from Theorem 2.11 that $L$ admits a 2-generator subgroup $G$ such that $\gamma_{2}(G)=\gamma_{2}(L)$, which is not possible as $\left|\gamma_{2}(L) / \gamma_{3}(L)\right|=p^{3}$. Hence $H=\gamma_{3}(L)$, and therefore, again by Theorem 2.11, $L$ admits a 3-generator subgroup $G$ of order $p^{7}$ such that $\gamma_{2}(G)=\gamma_{2}(L)$.

If $|L|=p^{8}$, then $L=\langle a, b, c, d\rangle$ such that $G=\langle a, b, c\rangle$ and $[d, G]=H$. If $|L| \geq p^{9}$, then, for some integer $k \geq 2, L=\left\langle a, b, c, x_{1}, \ldots, x_{k}\right\rangle$ such that $G=\langle a, b, c\rangle$ with $\gamma_{2}(G)=\gamma_{2}(L)$ and $\left[x_{i}, L\right]=H$ for $1 \leq i \leq k$. First assume that $\left[x_{i}, G\right]=H$ for some $1 \leq i \leq k$. Then the subgroup $M:=\langle a, b, c, d\rangle$, where $d=x_{i}$, is of order $p^{8}$ such that $[d, G]=H$ and $\gamma_{2}(M)=\gamma_{2}(L)$. Hence it is sufficient to work with $M$. So this case reduces to the preceding situation when $|L|=p^{8}$.

It now follows from the proof of Lemma 5.2 that we can modify the generating set for $G$ such that $\gamma_{2}(L)=\gamma_{2}(G)=\langle[a, b],[a, c],[b, c],[a,[a, b]]\rangle$ and $[c,[a, b]]=1$. Notice that $L / H$ is of nilpotency class 2 , and therefore $\gamma_{2}(L / H)=\langle[\bar{a}, \bar{b}],[\bar{a}, \bar{c}],[\bar{b}, \bar{c}]\rangle$, where $\bar{x}=x H$ for all $x \in L$. Also notice that $\bar{d} \in \mathrm{Z}(L / H)$.

If $[a, d]=[a,[a, b]]^{t}$ for some $t \in \mathbb{F}_{p}$, then $\left[a, d[a, b]^{-t}\right]=1$. Replacing $d$ by $d[a, b]^{-t}$, we can assume that $[a, d]=1$. If $[c, d] \neq 1$, then $[c, d]=[a,[a, b]]^{r}$ for some $r \in \mathbb{F}_{p}^{*}$. Let $\alpha_{i} \in \mathbb{F}_{p}$, $1 \leq i \leq 4$. If $\alpha_{1} \neq 0$, then modulo $H$ we can write

$$
[a, b]^{\alpha_{1}}[b, c]^{\alpha_{2}}[a, c]^{\alpha_{3}}=\left[a c^{-\alpha_{2} \alpha_{1}{ }^{-1}}, b^{\alpha_{1}} c^{\alpha_{3}}\right] .
$$

And

$$
[a,[a, b]]^{\alpha_{4}}=\left[a c^{-\alpha_{2} \alpha_{1}^{-1}},[a, b]^{\alpha_{4}}\right]
$$

If $\alpha_{1}=0$, then modulo $H$ we can write

$$
[b, c]^{\alpha_{2}}[a, c]^{\alpha_{3}}=\left[c, a^{-\alpha_{3}} b^{-\alpha_{2}}\right] .
$$


And

$$
[a,[a, b]]^{\alpha_{4}}=\left[c, d^{\alpha_{4} r^{-1}}\right] .
$$

Thus for $i \in \mathbb{F}_{p}$ and $\epsilon=0,1$, we get

$$
\gamma_{2}(L) / H=\bigcup_{\epsilon, i}\left[\bar{a}^{\epsilon} \bar{c}^{i}, \bar{L}\right]
$$

and

$$
H \subseteq \bigcap_{\epsilon, i}\left[a^{\epsilon} c^{i}, L\right],
$$

where $\bar{x}=x H$ for $x \in L$, and $i$ and $\epsilon$ are not simultaneously zero. Hence, by Lemma 2.5, $\mathrm{K}(L)=\gamma_{2}(L)$. Finally, if $[c, d]=1$, then $[b, d] \neq 1$. The assertion now follows on the same lines as above.

Now we consider the remaining case for the group $L$ with $|L| \geq p^{9}$, i.e., $\left[x_{i}, G\right]=1$ for all $1 \leq i \leq k$. Then $L$ is a central product of $G$ and $K:=\left\langle x_{1}, \ldots, x_{k}\right\rangle$ amalgamating $H$. Since $\mathrm{Z}(L) \leq \gamma_{2}(L), K$ can not be abelian. Thus $H=\left\langle\left[x_{i}, x_{j}\right]\right\rangle$ for some $1 \leq i<j \leq k$. Then the subgroup $N:=\langle a, b, c, d, e\rangle$, where $d=x_{i}$ and $e=x_{j}$, is of order $p^{9}$ such that $\gamma_{2}(N)=\gamma_{2}(L)$. Now on the lines of the preceding case, for $i \in \mathbb{F}_{p}$, it is not difficult to see that

$$
\gamma_{2}(N) / H=\bigcup_{i}\left[\bar{a} \bar{c}^{i} \bar{d}, \bar{N}\right] \bigcup[\bar{c} \bar{d}, \bar{N}]
$$

and

$$
H \subseteq \bigcap_{i}\left[a c^{i} d, N\right] \bigcap[c d, N]
$$

where $\bar{x}=x H$ for $x \in N$. Hence, again by Lemma 2.5, $\mathrm{K}(N)=\gamma_{2}(N)$.

Now assume that $\left|\gamma_{3}(L)\right|=p^{2}$. As in the above case, we can show that $H \not \subset \gamma_{3}(L)$. Then, by Theorem 2.11, either (i) $L$ admits a 2-generator subgroup $G$ of order $p^{5}$ and nilpotency class 3 such that $L$ is a central product of $G$ and subgroup $K$ with $\left|\gamma_{2}(K)\right|=p$ or (ii) $L$ admits a 3 -generator subgroup $G$ of order $p^{7}$ and nilpotency class 3 such that $\gamma_{2}(G)=\gamma_{2}(L)$ and $L$ is an amalgamated semidirect product of $G$ and a subgroup $K$ of nilpotency class at most 2. In case (i), it follows from Lemma 2.6 that $\mathrm{K}(L)=\gamma_{2}(L)$. So assume (ii).

If $|L|=p^{8}$, then $L=\langle a, b, c, d\rangle$ such that $G=\langle a, b, c\rangle$ and $\langle[d, G]\rangle=H$. If $|L| \geq p^{9}$, then, for some integer $k \geq 2, L=\left\langle a, b, c, x_{1}, \ldots, x_{k}\right\rangle$ such that $G=\langle a, b, c\rangle$ and $\left\langle\left[x_{i}, L\right]\right\rangle=H$ for $1 \leq i \leq k$. First assume that $\left[x_{i}, G\right]=H$ for some $1 \leq i \leq k$. Then the subgroup $M:=\langle a, b, c, d\rangle$, where $d=x_{i}$, is of order $p^{8}$ such that $[d, G]=H$ and $\gamma_{2}(M)=\gamma_{2}(L)$. As observed above, it is sufficient to work with the situation when $|L|=p^{8}$.

As explained in the proof of Lemma 5.2, we can assume that $H=\langle[b, c]\rangle$,

$$
\gamma_{3}(G)=\langle[a,[a, b]],[b,[a, b]]\rangle,
$$

$[a, c] \in \gamma_{3}(G)$ and $c \in \mathrm{C}_{G}\left(\gamma_{2}(G)\right)$. If $[b, d]=[b, c]^{t}$ for some $t \in \mathbb{F}_{p}$, then $\left[b, d c^{-t}\right]=1$. Set $d^{\prime}=d c^{-t}$. If $\left[c, d^{\prime}\right]=[b, c]^{s}$ for some $s \in \mathbb{F}_{p}$, then $\left[c, d^{\prime} b^{s}\right]=1$. Replacing $d$ by $d^{\prime} b^{s}$, we can assume that $[b, d]=[c, d]=1$. Let $[a, d]=[b, c]^{r}$ for some $r \in \mathbb{F}_{p}^{*}$. Let $\alpha_{1}, \alpha_{2}, \alpha_{3}, \alpha_{4} \in \mathbb{F}_{p}$. If $\alpha_{3} \neq 0$, then, modulo $H$ we can write

$$
[a, b]^{\alpha_{1}}[b,[a, b]]^{\alpha_{2}}[a,[a, b]]^{\alpha_{3}}=\left[a b^{\alpha_{2} \alpha_{3}{ }^{-1}}[a, b]^{\frac{1-\alpha_{1}}{2}}, b^{\alpha_{1}}[a, b]^{\alpha_{3}}\right] .
$$

And

$$
[b, c]^{\alpha_{4}}=\left[a b^{\alpha_{2} \alpha_{3}-1}[a, b]^{\frac{1-\alpha_{1}}{2}}, d^{\alpha_{4} r^{-1}}\right] .
$$


If $\alpha_{3}=0$, then modulo $H$ we can write

$$
[a, b]^{\alpha_{1}}[b,[a, b]]^{\alpha_{2}}=\left[b[a, b]^{-\frac{\alpha_{1}+1}{2}}, a^{-\alpha_{1}}[a, b]^{\alpha_{2}}\right] .
$$

And

$$
[b, c]^{\alpha_{4}}=\left[b[a, b]^{-\frac{\alpha_{1}+1}{2}}, c^{\alpha_{4}}\right] .
$$

Thus, for $i, j \in \mathbb{F}_{p}$ and $\epsilon=0,1$ such that $i$ and $\epsilon$ are not simultaneously zero, we get

$$
\gamma_{2}(L) / H=\bigcup_{\epsilon, i, j}\left[\bar{a}^{\epsilon} \bar{b}^{i}[a, b]^{j}, \bar{L}\right]
$$

and

$$
H \subseteq \bigcap_{\epsilon, i, j}\left[a^{\epsilon} b^{i}[a, b]^{j}, L\right],
$$

where $\bar{x}=x H$ for $x \in L$. Hence $\mathrm{K}(L)=\gamma_{2}(L)$.

Finally assume that $\left[x_{i}, G\right]=1$ for all $1 \leq i \leq k$. In this case, $L$ is a central product of $G$ and $K:=\left\langle x_{1}, \ldots, x_{k}\right\rangle$ amalgamating some subgroup containing $H$. Since $\mathrm{Z}(L) \leq \gamma_{2}(L), K$ can not be abelian. Thus $H=\left\langle\left[x_{i}, x_{j}\right]\right\rangle$ for some $1 \leq i<j \leq k$. Then the subgroup $N:=\langle a, b, c, d, e\rangle$, where $d=x_{i}$ and $e=x_{j}$, is of order $p^{9}$ such that $\gamma_{2}(N)=\gamma_{2}(L)$. For $i \in \mathbb{F}_{p}$, it is again easy to see that

and

$$
\gamma_{2}(N) / H=\bigcup_{i}\left[\bar{a} \bar{b}^{i} \bar{d}[\bar{a}, \bar{b}]^{\frac{1-\alpha_{1}}{2}}, \bar{N}\right] \bigcup\left[\bar{b} \bar{d}[\bar{a}, \bar{b}]^{-\frac{\alpha_{1}+1}{2}}, \bar{N}\right]
$$

$$
H \subseteq \bigcap_{i}\left[a b^{i} d[a, b]^{\frac{1-\alpha_{1}}{2}}, N\right] \bigcap\left[b d[a, b]^{-\frac{\alpha_{1}+1}{2}}, N\right],
$$

where $\bar{x}=x H$ for $x \in N$. Thus $\mathrm{K}(N)=\gamma_{2}(N)$, and the proof is complete.

We are now ready to prove Theorem A.

Proof of Theorem $A$. Let $G$ be a finite $p$-groups such that $\gamma_{2}(G)$ is of order $p^{4}$ and exponent $p$. Also let $\mathrm{Z}(G) \leq \gamma_{2}(G)$. Notice that the nilpotency class of $G$ is at most 5 . By Remark 2.8 we have $b(G) \geq 3$. Notice that $|G| \geq p^{6}$. If $b(G)=4$, then $\mathrm{K}(G)=\gamma_{2}(G)$. So we assume that $b(G)=3$. If the nilpotency class of $G$ is at most 3, then $\gamma_{2}(G)$ is abelian. When the nilpotency class of $G$ is 2, the assertion follows from Lemmas 3.1-3.4. Now let the nilpotency class of $G$ be 3. It follows from 14 that there is no group of order $p^{6}$ and nilpotency class 3 satisfying the given hypothesis. Hence $|G| \geq p^{7}$. If $|\mathrm{Z}(G)| \leq p^{2}$, then $\mathrm{K}(G)=\gamma_{2}(G)$ by Lemma 5.2, If $|G| \geq p^{8}$ and $|\mathrm{Z}(G)|=p^{3}$, then by Lemma 6.1 we have $\mathrm{K}(G)=\gamma_{2}(G)$. Finally if $|G|=p^{7}$ and $|\mathrm{Z}(G)|=p^{3}$, then by Lemma 5.2 we have $\mathrm{K}(G) \neq \gamma_{2}(G)$. It only remains to handle the cases when the nilpotency class of $G$ is 4 or 5 .

Let the nilpotency class of $G$ be 4 and $b(G)=3$. If $|G|=p^{6}$, then it follows from Lemma 4.1 that $\mathrm{K}(G)=\gamma_{2}(G)$ if and only if $|\mathrm{Z}(G)|=p$. So assume that $|G| \geq p^{7}$. By Lemma 2.4 we have $|\mathrm{Z}(G)| \leq p^{2}$. It follows from Theorem 2.10 that $G$ admits a normal subgroup $H$ of order $p$ such that $|G / H: \mathrm{Z}(G / H)|=p^{3}$. The only choice for $H$, in this case, is $\gamma_{4}(G)$. If not, then $G / H$ will be of nilpotency class 4 , which is not possible as $(G / H) / \mathrm{Z}(G / H)$ can have nilpotency class at most 2. Thus $G / H$ has nilpotency class 3. For the existence of such a group $G$, it is necessary that $\left(\gamma_{2}(G) / H\right) \cap \mathrm{Z}(G / H)$ is of order $p^{2}$. Hence there exists a 2-generator subgroup $S$ of $G$ such that $\gamma_{2}(G)=\gamma_{2}(S)$. Since $\gamma_{2}(S) / \gamma_{3}(S)$ is cyclic and $\left[\gamma_{2}(S), \gamma_{3}(S)\right]=1$, it follows that $\gamma_{2}(S)$ is abelian. Hence $\gamma_{2}(G)$ is abelian. Now invoking Theorem 2.11 there exists a 2-generator 
subgroup $T$ of $G$ such that $|T|=p^{6}, \gamma_{2}(T)=\gamma_{2}(G)$ and $G$ is an amalgamated semidirect product of $T$ and a subgroup $K$ with $\left|\gamma_{2}(K)\right| \leq p$. Also $K=\left\langle x_{1}, \ldots, x_{k}\right\rangle$, for some $k \geq 1$ such that $\left[x_{i}, L\right]=H$ for $1 \leq i \leq k$. If $\left[x_{i}, T\right]=H$ for some $1 \leq i \leq k$, then the subgroup $M:=\langle a, b, c\rangle$, where $c=x_{i}$ is of order $p^{7}$ such that $\gamma_{2}(M)=\gamma_{2}(G)$. Hence it follows from Lemma 4.2 that $\mathrm{K}(G)=\gamma_{2}(G)$. Now assume that $\left[x_{i}, T\right]=1$ for all $1 \leq i \leq k$. Since $\mathrm{Z}(G) \leq \gamma_{2}(G)$, it follows that $K$ is non-abelian. Thus $G$ is a central product of $T$ and $K$ amalgamating a subgroup containing $H$. If $|\mathrm{Z}(G)|=p$, then $|\mathrm{Z}(T)|=p$, and therefore by Lemma 4.1 we have $\mathrm{K}(T)=\gamma_{2}(T)$. Hence $\mathrm{K}(G)=\gamma_{2}(G)$. Now we take the case when $|\mathrm{Z}(G)|=p^{2}$. Notice that $\gamma_{2}(K)=H$, and therefore there exists $x_{i}, x_{j} \in K$ such that $H=\left\langle\left[x_{i}, x_{j}\right]\right\rangle$ for some $1 \leq i<j \leq k$. Let $N:=\langle a, b, c, d\rangle$, where $c=x_{i}$ and $d=x_{j}$. Then $\gamma_{2}(G)=\gamma_{2}(N)$. Set $\bar{N}=N / H$. As observed in the proof of Lemma 4.2

$$
\gamma_{2}(\bar{N})=\langle[\bar{a}, \bar{b}],[\bar{a},[\bar{a}, \bar{b}]],[\bar{b},[\bar{a}, \bar{b}]]\rangle .
$$

It is now not difficult to see that for $i, j \in \mathbb{F}_{p}$ we have

$$
\gamma_{2}(\bar{N})=\bigcup_{i, j}\left[\bar{a}^{i} \bar{b}^{j} \bar{c}, \bar{N}\right]
$$

An easy computation also shows that $H \subseteq\left[a^{i} b^{j} c, N\right]$ for all $i, j \in \mathbb{F}_{p}$. Hence $\mathrm{K}(N)=\gamma_{2}(N)$ by Lemma 2.5, and therefore we have $\mathrm{K}(G)=\gamma_{2}(G)$.

Finally let the nilpotency class of $G$ be 5 . We claim that $b(G)=4$, and hence we are done. Contrarily assume that $b(G)=3$. Since the nilpotency class of $G / \mathrm{Z}(G)$ is 4 and $\mathrm{Z}(G) \leq \gamma_{2}(G)$, we have $|\mathrm{Z}(G)|=p$. Hence, in view of Theorem 2.10, the only choice for normal subgroup $H$ of $G$ such that $|G / H: \mathrm{Z}(G / H)|=p^{3}$ is $\mathrm{Z}(G)$. Thus $\left|G / \mathrm{Z}(G): \mathrm{Z}_{2}(G) / \mathrm{Z}(G)\right|=p^{3}$, which implies that $\left|G / \mathrm{Z}_{2}(G)\right|=p^{3}$, where $\mathrm{Z}_{2}(G)$ denotes the second center of $G$. This contradicts the fact that the nilpotency class of $G / \mathrm{Z}_{2}(G)$ is 3 . Our claim is now settled, and the proof of the theorem is complete.

\section{2-Groups AND PROOF OF THeOREM B}

We start with the following result of Wilkens [22] on 2-group of breadth 3 .

Theorem 7.1. Let $P$ be a finite 2-group of breadth 3 . Then one of the following holds:

(i) $\left|\gamma_{2}(P)\right| \leq 2^{3}$.

(ii) $|P: \mathrm{Z}(P)| \leq 2^{4}$.

(iii) $\left|\gamma_{2}(P)\right|=2^{4}$ and there is $R, R \leq \Omega_{1}(\mathrm{Z}(P)),|R|=2$, such that $|P / R: \mathrm{Z}(P / R)| \leq 2^{3}$.

(iv) $\left|\gamma_{2}(P)\right|=2^{4}$ and $P$ is a central product $G \mathrm{C}_{P}(G)$, with $\mathrm{C}_{P}(G)$ is abelian and $G$ lies in one of the following five classes of groups:

(1) There are $i, j \in \mathbb{N}$ with $G \cong \hat{G} /\left\langle x^{4 i}, y^{4 j}\right\rangle$, where $\gamma_{4}(\hat{G})=1=\mho_{2}\left(\gamma_{2}(\hat{G})\right)=\mho_{1}\left(\gamma_{3}(\hat{G})\right)$, and $\hat{G}$ is free in the category of these groups.

(2) There are $i, j \in \mathbb{N}$ with $G \cong \hat{G} /\left\langle x^{4 i}, y^{2 j}\right\rangle$, where

$$
\hat{G}=\left\langle x, y \mid[x, y]^{y}=[y, x],\left[y,{ }_{2} x\right]^{2}=1=\left[y,{ }_{3} x\right]^{2}=\left[y,{ }_{2} x, y\right]=\left[y,{ }_{3} x, y\right]=\left[y,{ }_{4} x\right]\right\rangle .
$$

(3) There are $i, j, k \in \mathbb{N}$ with $G \cong \hat{G} /\left\langle x^{4 i}, y^{2 j}, z^{2 k}\right\rangle, \hat{G}=\langle x, y, z\rangle$ has $\gamma_{4}(G)=1=\mho_{1}\left(\gamma_{3}(G)\right)$, and, apart from that, is defined by the relations

$$
[x, y]^{4}=1=[x, y]^{2}[x, y, y]=[x, y, z]=[x, z]^{2}=[x, z, z]=[x, z, y]=[x, z, x]=[y, z] .
$$


(4) There are $i, j, k, l \in \mathbb{N}$ with $G \cong \hat{G} /\left\langle x^{2 i}, y^{2 j}, a^{2 k}, t^{2 l}\right\rangle, \hat{G}=\langle a, t, x, y\rangle$ of nilpotency class 3 with additional relations $[x, a]^{2}=1=[x, a, w]=[y, t, w]=[y, t]^{2}, w \in\{a, t, x, y\},[x, t]=$ $[y, t][y, a]=1,[x, y]^{4}=1=[x, y, a]=[x, y, t]=[x, y, y][x, y]^{2}=[x, y, x][x, y]^{2},[t, a] \in\left\langle[x, y]^{2}\right\rangle$.

(5) There are $i, j, k, l, m \in \mathbb{N}$ such that $G=\hat{G} /\left\langle x^{2 i}, v^{2 j}, v_{1}{ }^{2 k}, v_{2}{ }^{2 l}, v_{3}{ }^{2 m}\right\rangle$, and $\hat{G}=\left\langle a, v, v_{1}, v_{2}, v_{3}\right\rangle$ is of nilpotency class 2 with $\Phi(\hat{G}) \leq \mathrm{Z}(\hat{G})$ and is otherwise defined by $\left[v_{2}, x\right]=1=\left[v_{1}, v\right]=$ $\left[v_{3}, x\right]\left[v_{3}, v\right],\left[v_{i}, v_{j}\right] \leq\left\langle\left[v_{3}, x\right]\right\rangle$.

The following result reduces our study to 2-groups of nilpotency class 2 and 3 , and to groups of order $2^{7}$ if the nilpotency class is 3 .

Theorem 7.2. Let $P$ be a finite 2-group such that $\mathrm{Z}(P) \leq \gamma_{2}(P), \gamma_{2}(P)$ is elementary abelian of order $2^{4}$ and $b(P)=3$. Then

(i) The nilpotency class of $P$ is either 2 or 3.

(ii) If the nilpotency class of $P$ is 2 , then $|P| \geq 2^{8}$.

(iii) If the nilpotency class of $P$ is 3 , then there exists a 3-generator subgroup $G$ of $P$ having the same nilpotency class as that of $P$ such that $\gamma_{2}(G)=\gamma_{2}(P)$. Moreover, $|G|=2^{7}$, and if $|P| \geq 2^{8}$, then $P$ is an amalgamated semidirect product of $G$ and a subgroup $K$ with $\left|\gamma_{2}(K)\right|=2$. If $K$ is non-abelian, then it is isoclinic to an extraspecial 2-group.

Proof. For the given group $P$, one of the assertions (ii) - (iv) of Theorem 7.1 holds true. We start by noting that $|P| \geq 2^{7}$. Also, if Theorem 7.1 (iv) holds, then $P$ is isomorphic to a group in class (5) of Theorem 7.1(iv), which consists of groups of nilpotency class 2. So we only need to take into consideration the assertions (ii) - (iii) of Theorem 7.1. Let the nilpotency class of $P$ be at least 4. Then, by Lemma 2.4 $\mathrm{Z}(P)$ can not be maximal in $\gamma_{2}(P)$. So in the case when Theorem 7.1(ii) holds, we must get $\left|\gamma_{2}(P) / \mathrm{Z}(P)\right| \geq 4$. Since $P$ is non-abelian, it follows that $|P| \leq 2^{6}$, which is not possible as observed above. Now assume that Theorem 7.1 (iii) holds. Then there exists a central subgroup $R$ of order 2 such that $|P / R: \mathrm{Z}(P / R)| \leq 8$. Also the nilpotency class of $P / R$ is at least 3. This is possible only when $\left|\gamma_{2}(P / R): \gamma_{2}(P / R) \cap \mathrm{Z}(P / R)\right|=2$, which, by Lemma 2.4. implies that the nilpotency class of $P / R$ is 3. Thus $(P / R) / Z(P / R)$ is non-abelian, which is not possible as shown in the next para. Hence the nilpotency class of $P$ is either 2 or 3. Let the nilpotency class of $P$ be 2 and $|P|=2^{7}$. Then $P$ is generated by at most three elements, which is not possible. Hence $|P| \geq 2^{8}$ in this case.

Now we assume that the nilpotency class of $P$ is 3. If Theorem[7.1(ii) holds, then, by the given hypotheses, it follows that $\left|\gamma_{2}(P) / \mathrm{Z}(P)\right|=2$. Hence $P$ itself is a 3-generator group of order $2^{7}$. Next assume that Theorem 7.1(iii) holds. Thus there exists a central subgroup $R$ of order 2 such that $|P / R: \mathrm{Z}(P / R)| \leq 8$. In our case, it is easy to deduce that $|P / R: \mathrm{Z}(P / R)|=8$. Set $\bar{P}=P / R$. We claim that $\bar{P} / \mathrm{Z}(\bar{P})$ is abelian. Contrarily assume that $\bar{P} / \mathrm{Z}(\bar{P})$ is non-abelian. Then the nilpotency class of $\bar{P}$ is 3 and $\bar{P}=\langle\bar{a}, \bar{b}, \mathrm{Z}(\bar{P})\rangle$ for some $a, b \in P$, where $\bar{x}$ denotes $x R$ for $x \in P$. Since the exponent of $\bar{P} / \mathrm{Z}(\bar{P})$ can not be 2 , it follows that $[\bar{a}, \bar{b}]=\left(\bar{a}^{\epsilon_{1}} \bar{b}^{\epsilon_{2}}\right)^{2}$ modulo $\mathrm{Z}(\bar{P})$, where $\epsilon_{i} \in \mathbb{F}_{2}$. Hence $\mathrm{C}_{\bar{P}}([\bar{a}, \bar{b}])$ is maximal in $\bar{P}$. This implies that $[[\bar{a}, \bar{b}], \bar{P}]$ is of order 2 , which contradicts the fact that $\left|\gamma_{2}(\bar{P})\right|=8$. The claim is now settled. Thus the nilpotency class of $\bar{P}$ is 2 . If the exponent of $\bar{P} / \mathrm{Z}(\bar{P})$ is 4 , then $\bar{P}=\langle\bar{a}, \bar{b}, \mathrm{Z}(\bar{P})\rangle$ for some $a, b \in P$. This implies that $\left|\gamma_{2}(\bar{P})\right|=2$, which again contradicts the fact that $\left|\gamma_{2}(\bar{P})\right|=8$. Hence the exponent of $\bar{P} / \mathrm{Z}(\bar{P})$ is 2 , and therefore $\bar{P}=\langle\bar{a}, \bar{b}, \bar{c}, \mathrm{Z}(\bar{P})\rangle$ for some $a, b, c \in P$. 
Let $G:=\langle a, b, c\rangle$. As proved in the reduction theorem for $p$ odd case, $\gamma_{2}(G)=\gamma_{2}(P)$, and therefore $|G|=2^{7}$. Let $|P| \geq 2^{8}$. Then $P=\left\langle a, b, c, x_{1}, \ldots, x_{k}\right\rangle$ for some integer $k \geq 1$. Let $K:=\left\langle x_{1}, \ldots, x_{k}\right\rangle$. Then $\left[x_{i}, K\right]=R$. It now follows that $G$ and $K$ are the desired subgroups, which completes the proof.

We deduce the following result using GAP [3]. A theoretical proof goes on the lines of Lemma 5.1

Lemma 7.3. Let $G$ be a group of order $2^{7}$ with $b(G)=3, \mathrm{Z}(G) \leq \gamma_{2}(G)$ and $\gamma_{2}(G)$ elementary abelian of order $2^{4}$. Then $\mathrm{K}(G)=\gamma_{2}(G)$.

We again use GAP to establish the following two lemmas, whose theoretical proofs can be written on the lines of corresponding results of Section 3 ,

Lemma 7.4. Let $G$ be a finite p-group of order $2^{8}$ and nilpotency class 2 such that $\gamma_{2}(G)$ is elementary abelian of order $2^{4}$. If $G$ is not of conjugate type $\left\{1,2^{3}\right\}$ or $\left\{1,2^{2}, 2^{3}\right\}$, then $\mathrm{K}(G) \neq \gamma_{2}(G)$. Moreover, every element of $\gamma_{2}(G)$ can be written as a product of at most two elements from $\mathrm{K}(G)$.

Lemma 7.5. Let $G$ be a finite 2 -group of order $2^{8}$, nilpotency class 2 and conjugate type $\left\{1,2^{3}\right\}$ such that $\gamma_{2}(G)$ is elementary abelian of order $2^{4}$. Then $\mathrm{K}(G)=\gamma_{2}(G)$.

The proof of the following lemma goes on the lines of the proof of Lemma 3.2. Since the proof for $p=2$ is a bit different towards the end, we have decided to include a part of the proof here too.

Lemma 7.6. Let $G$ be a finite 2-group of order $2^{8}$, nilpotency class 2 and conjugate type $\left\{1,2^{2}, 2^{3}\right\}$ such that $\gamma_{2}(G)$ is elementary abelian of order $2^{4}$. Then $\mathrm{K}(G)=\gamma_{2}(G)$ if and only if $G$ admits a generating set $\{a, b, c, d\}$ such that $[a, b]=1=[c, d]$. Moreover, if $\mathrm{K}(G) \neq \gamma_{2}(G)$, then every element of $\gamma_{2}(G)$ can be written as a product of at most two elements from $\mathrm{K}(G)$.

Proof. The 'if' part is the same as that of Lemma 3.2. For 'only if' part, as is also done in Lemma 3.2, we provide a contrapositive proof. We assume that $G$ admits no generating set $\left\{x_{1}, x_{2}, x_{3}, x_{4}\right\}$ such that $\left[x_{1}, x_{2}\right]=1=\left[x_{3}, x_{4}\right]$. By the given hypothesis, we can always choose a generating set $\{a, b, c, d\}$ for $G$ such that $[a, c]=1$ and none of the other basic commutators of weight two in generators is trivial. So we can assume that

$$
[c, d]=[a, b]^{t_{1}}[b, c]^{t_{2}}[b, d]^{t_{3}}[a, d]^{t_{4}},
$$

where $t_{i} \in \mathbb{F}_{2}$ and $1 \leq i \leq 4$, and therefore $\gamma_{2}(G)=\langle[a, b],[b, c],[b, d],[a, d]\rangle$.

We can write the preceding equation as

$$
\left[b, a^{-t_{1}} c^{t_{2}} d^{t_{3}}\right]\left[d, a^{-t_{4}} c\right]=1 .
$$

We claim that $t_{3}=0$. If $t_{3}=1$, then replacing $d$ by $d^{\prime}:=a^{-t_{1}} c^{t_{2}} d$ and a proper substitution reduces (7.1) to $\left[d^{\prime}, b^{-1} a^{-t_{4}} c\right]=1$. Now replacing $b$ by $b^{\prime}:=b^{-1} a^{-t_{4}} c$, we get a generating set $\left\{a, b^{\prime}, c, d^{\prime}\right\}$ for $G$ such that $\gamma_{2}(G)=\left\langle\left[a, b^{\prime}\right],\left[b^{\prime}, c\right],\left[b^{\prime}, d^{\prime}\right],\left[c, d^{\prime}\right]\right\rangle$ and $[a, c]=1=\left[b^{\prime}, d^{\prime}\right]$, which contradicts our hypothesis. Our claim is now settled. 
So now onwards we assume that $t_{3}=0$. Hence (7.1) reduces to $\left[b, a^{-t_{1}} c^{t_{2}}\right]\left[d, c a^{-t_{4}}\right]=1$. Now replace $c$ by $c^{\prime}:=c a^{-t_{4}}$. A simple computation gives

$$
\left[b, a^{-t_{1}} c^{t_{2}}\right]=\left[b, a^{-t_{1}}\left(c^{\prime} a^{t_{4}}\right)^{t_{2}}\right]=\left[b, a^{t_{4} t_{2}-t_{1}} c^{\prime t_{2}}\right] .
$$

Thus (7.1) reduces to

$$
\left[b, a^{t_{4} t_{2}-t_{1}} c^{t_{2}}\right]\left[d, c^{\prime}\right]=1 .
$$

We claim that $t_{4} t_{2}-t_{1} \neq 0$. Contrarily assume that $t_{4} t_{2}-t_{1}=0$. Then (7.2) takes the form $\left[b, c^{\prime t_{2}}\right]\left[d, c^{\prime}\right]=1$, which gives $\left[b^{t_{2}} d, c^{\prime}\right]=1$. Now replacing $d$ by $d^{\prime}:=b^{t_{2}} d$, we get a generating set $\left\{a, b, c^{\prime}, d^{\prime}\right\}$ of $G$ such that $\left[a, c^{\prime}\right]=1=\left[d^{\prime}, c^{\prime}\right]$, which gives that the size of the conjugacy class of $c^{\prime}$ in $G$ is 2, a contradiction to the given hypothesis. This settles our claim.

So we now assume $t_{4} t_{2}-t_{1} \neq 0$. Then replacing $a$ by $a^{\prime}:=a^{t_{4} t_{2}-t_{1}} c^{\prime t_{2}}$, we get a new generating set $\left\{a^{\prime}, b, c^{\prime}, d\right\}$ such that $\gamma_{2}(G)=\left\langle\left[a^{\prime}, b\right],\left[b, c^{\prime}\right],[b, d],\left[a^{\prime}, d\right]\right\rangle,\left[a^{\prime}, c^{\prime}\right]=1$ and, by (7.2), $\left[a^{\prime}, b\right]=\left[c^{\prime}, d\right]$. Now we claim that $\left[a^{\prime}, b\right]\left[b, c^{\prime}\right]\left[a^{\prime}, d\right] \notin \mathrm{K}(G)$. Contrarily assume that $\left[a^{\prime}, b\right]\left[b, c^{\prime}\right]\left[a^{\prime}, d\right] \in \mathrm{K}(G)$. Thus

$$
\left[a^{\prime}, b\right]\left[b, c^{\prime}\right]\left[a^{\prime}, d\right]=\left[a^{\prime \alpha_{1}} b^{\alpha_{2}} c^{\alpha_{3}} d^{\alpha_{4}}, a^{\prime \beta_{1}} b^{\beta_{2}} c^{\prime \beta_{3}} d^{\beta_{4}}\right],
$$

for some $\alpha_{i}, \beta_{j} \in \mathbb{F}_{2}, 1 \leq i, j \leq 4$. Expanding the right hand side and comparing powers both side, we get

$$
\begin{aligned}
\alpha_{1} \beta_{4}+\alpha_{4} \beta_{1} & =1, \\
\alpha_{2} \beta_{4}+\alpha_{4} \beta_{2} & =0, \\
\alpha_{2} \beta_{3}+\alpha_{3} \beta_{2} & =1, \\
\alpha_{1} \beta_{2}+\alpha_{2} \beta_{1}+\alpha_{3} \beta_{4}+\alpha_{4} \beta_{3} & =1 .
\end{aligned}
$$

First assume that $\alpha_{2}=0$. Then from (7.5) we get $\alpha_{3}=\beta_{2}=1$, and hence from (7.4) we get $\alpha_{4}=0$, which using(7.3) gives $\alpha_{1}=\beta_{4}=1$. But these values contradict (7.6). So assume that $\alpha_{2}=1$. If $\beta_{2}=0$, then (7.4) gives $\beta_{4}=0$ and (7.5) gives $\beta_{3}=1$. Substituting $\beta_{4}=0$ in (7.3) we get $\alpha_{4}=\beta_{1}=1$, which contradict (7.6). Hence $\beta_{2} \neq 0$.

Finally assume that $\alpha_{2}=\beta_{2}=1$. Therefore above equations reduces to

$$
\begin{aligned}
\alpha_{1} \beta_{4}+\alpha_{4} \beta_{1} & =1, \\
\beta_{4}+\alpha_{4} & =0, \\
\beta_{3}+\alpha_{3} & =1, \\
\alpha_{1}+\beta_{1}+\alpha_{3} \beta_{4}+\alpha_{4} \beta_{3} & =1 .
\end{aligned}
$$

As, by (7.8), $\alpha_{4}=\beta_{4}$, from (7.7) we get $\alpha_{4}=1=\alpha_{1}+\beta_{1}$. Now putting $\alpha_{4}=\beta_{4}=1$ in (7.10) and using (7.9), we get $\alpha_{1}+\beta_{1}=0$, which is not possible. Hence $\left[a^{\prime}, b\right]\left[b, c^{\prime}\right]\left[a^{\prime}, d\right] \notin \mathrm{K}(G)$. The final assertion follows the similar way as in Lemma 3.2. This completes the proof.

The proof of the following lemma goes on the lines of the proof of Lemma 3.4 .

Lemma 7.7. Let $G$ be a finite 2-group of nilpotency class 2 and order at least $2^{9}$ such that $\mathrm{Z}(G)=\gamma_{2}(G)$, Theorem 7.1)(iii) holds and $\gamma_{2}(G)$ is elementary abelian of order $2^{4}$. Then $\mathrm{K}(G)=\gamma_{2}(G)$. 
Let $P$ be a 2-group of breadth 3 which satisfies Theorem 7.1 (iv) and $\gamma_{2}(P)$ be elementary abelian of order 16. Then, by a careful inspection, it follows from Theorem 7.1 that $P$ is isoclinic to the group $T$ presented as

$$
\begin{aligned}
T= & \left\langle v_{1}, v_{2}, v_{3}, v_{4}, v_{5}\right|\left[v_{4}, v_{2}\right]=\left[v_{5}, v_{1}\right]=1,\left[v_{1}, v_{2}\right]=\left[v_{3}, v_{4}\right]^{r},\left[v_{2}, v_{3}\right]=\left[v_{3}, v_{4}\right]^{s} \\
& {\left[v_{3}, v_{1}\right]=\left[v_{3}, v_{4}\right]^{t},\left[v_{3}, v_{4}\right]=\left[v_{3}, v_{5}\right], v_{i}^{2 k_{i}}=1,\left[v_{i}^{2}, v_{j}\right]=1(1 \leq i, j \leq 5) } \\
& {\left.[x, y, z]=1 \text { for all } x, y, z \in\left\{v_{1}, \ldots, v_{5}\right\}\right\rangle }
\end{aligned}
$$

for some positive integers $k_{i}$ 's and and some $r, s, t \in \mathbb{F}_{2}$.

Lemma 7.8. Let $G$ be a finite 2-group of breadth 3 such that Theorem 7.1(iv) holds, $\mathrm{Z}(G) \leq$ $\gamma_{2}(G)$ and $\gamma_{2}(G)$ is elementary abelian of order $2^{4}$. Then $G$ is isoclinic to the group $T$ given by (7.11) for some $r, s, t \in \mathbb{F}_{2}$ and $k_{i}=1,1 \leq i \leq 5$, and $\mathrm{K}(G) \neq \gamma_{2}(G)$ if and only if $r=s=t=0$.

Proof. It is not difficult to see that $G$ is isoclinic to the group $T$ given by (7.11) for $k_{i}=1$, $1 \leq i \leq 5$ and some $r, s, t \in \mathbb{F}_{2}$. We now prove the second assertion. First assume that $r=s=t=0$. We claim that $\left[v_{4}, v_{1}\right]\left[v_{4}, v_{3}\right]\left[v_{5}, v_{2}\right] \notin \mathrm{K}(G)$. Otherwise, there exist $\alpha_{i}, \beta_{i} \in \mathbb{F}_{2}$ such that

$$
\left[v_{4}, v_{1}\right]\left[v_{4}, v_{3}\right]\left[v_{5}, v_{2}\right]=\left[v_{4}^{\alpha_{1}} v_{5}^{\alpha_{2}} v_{1}^{\alpha_{3}} v_{2}^{\alpha_{4}} v_{3}^{\alpha_{5}}, v_{4}^{\beta_{1}} v_{5}^{\beta_{2}} v_{1}^{\beta_{3}} v_{2}^{\beta_{4}} v_{3}^{\beta_{5}}\right] .
$$

Expanding right side and comparing the powers of commutators, we get

$$
\begin{aligned}
\alpha_{1} \beta_{2}+\alpha_{2} \beta_{1} & =0, \\
\alpha_{1} \beta_{3}+\alpha_{3} \beta_{1} & =1, \\
\alpha_{2} \beta_{4}+\alpha_{4} \beta_{2} & =1, \\
\alpha_{1} \beta_{5}+\alpha_{5} \beta_{1}+\alpha_{2} \beta_{5}+\alpha_{5} \beta_{2} & =1 .
\end{aligned}
$$

First assume that $\alpha_{1}=0$. Then (7.13) gives $\alpha_{3}=\beta_{1}=1$, hence by (7.12) we get $\alpha_{2}=0$, which gives $\alpha_{4}=\beta_{2}=1$. But these values contradict (7.15). So now assume that $\alpha_{1}=1$. If $\beta_{1}=0$, then by (7.12) and (7.13) we get $\beta_{2}=0$ and $\beta_{3}=1$, respectively. As $\beta_{2}=0$, by (7.14) we get $\alpha_{2}=\beta_{4}=1$; but these values contradict (7.15). Finally assume that $\alpha_{1}=$ $\beta_{1}=1$. Then by (7.12) and (7.14) we get $\alpha_{2}=\beta_{2}=1$, which again contradict (7.15). Hence $\left[v_{4}, v_{1}\right]\left[v_{4}, v_{3}\right]\left[v_{5}, v_{2}\right] \notin \mathrm{K}(G)$.

Conversely, assume that at least one of $r, s, t$ is non-zero. We'll show that $\mathrm{K}(G)=\gamma_{2}(G)$. It is easy to see that except $\left[v_{4}, v_{1}\right]\left[v_{4}, v_{3}\right]\left[v_{5}, v_{2}\right],\left[v_{4}, v\right]\left[v_{4}, v_{1}\right]\left[v_{4}, v_{3}\right]\left[v_{5}, v_{2}\right]$, all elements of $\gamma_{2}(G)$ lie in $\mathrm{K}(G)$. We'll first show that $\left[v_{4}, v_{1}\right]\left[v_{4}, v_{3}\right]\left[v_{5}, v_{2}\right] \in \mathrm{K}(G)$. If $r=1$, then $\left[v_{1}, v_{2}\right]=\left[v_{3}, v_{4}\right]=$ $\left[v_{4}, v_{3}\right]$, and therefore

$$
\left[v_{4}, v_{1}\right]\left[v_{5}, v_{2}\right]\left[v_{4}, v_{3}\right]=\left[v_{4}, v_{1}\right]\left[v_{5}, v_{2}\right]\left[v_{1}, v_{2}\right]=\left[v_{4} v_{5} v_{1}, v_{1} v_{2}\right] .
$$

So let $r=0$. If $t=1$, then for any value of $s$ we have

$$
\left[v_{4} v_{5} v_{1}, v_{1} v_{2} v_{3}\right]=\left[v_{4}, v_{1}\right]\left[v_{4}, v_{3}\right]\left[v_{5}, v_{2}\right]\left[v_{5}, v_{3}\right]\left[v_{1}, v_{3}\right]=\left[v_{4}, v_{1}\right]\left[v_{4}, v_{3}\right]\left[v_{5}, v_{2}\right] \text {. }
$$

If $t=0$ and $s=1$, then

$$
\left[v_{4} v_{5} v_{2}, v_{1} v_{2} v_{3}\right]=\left[v_{4}, v_{1}\right]\left[v_{4}, v_{3}\right]\left[v_{5}, v_{2}\right]\left[v_{5}, v_{3}\right]\left[v_{2}, v_{3}\right]=\left[v_{4}, v_{1}\right]\left[v_{4}, v_{3}\right]\left[v_{5}, v_{2}\right] .
$$

Now we take $\left[v_{4}, v_{5}\right]\left[v_{4}, v_{1}\right]\left[v_{4}, v_{3}\right]\left[v_{5}, v_{2}\right]$. First let $r=1$. Then

$$
\left[v_{4} v_{5} v_{1}, v_{5} v_{1} v_{2}\right]=\left[v_{4}, v_{5}\right]\left[v_{4}, v_{1}\right]\left[v_{5}, v_{2}\right]\left[v_{1}, v_{2}\right] \text {. }
$$


Next let $r=0$. If $t=1$, then

$$
\left[v_{4} v_{5} v_{1}, v_{5} v_{1} v_{2} v_{3}\right]=\left[v_{4}, v_{5}\right]\left[v_{4}, v_{1}\right]\left[v_{4}, v_{3}\right]\left[v_{5}, v_{2}\right]\left[v_{5}, v_{3}\right]\left[v_{1}, v_{3}\right]=\left[v_{4}, v_{5}\right]\left[v_{4}, v_{1}\right]\left[v_{4}, v_{3}\right]\left[v_{5}, v_{2}\right]
$$

If $t=0$ and $s=1$, then we finally get

$$
\left[v_{4} v_{1} v_{2}, v_{5} v_{1} v_{3}\right]=\left[v_{4}, v_{5}\right]\left[v_{4}, v_{1}\right]\left[v_{4}, v_{3}\right]\left[v_{1}, v_{3}\right]\left[v_{2}, v_{5}\right]=\left[v_{4}, v_{5}\right]\left[v_{4}, v_{1}\right]\left[v_{4}, v_{3}\right]\left[v_{5}, v_{2}\right] .
$$

Hence $\left[v_{4}, v_{5}\right]\left[v_{4}, v_{1}\right]\left[v_{4}, v_{3}\right]\left[v_{5}, v_{2}\right] \in \mathrm{K}(G)$, and the proof of the lemma is now complete.

We can now write a proof of Theorem B.

Proof of Theorem B. Let $G$ be a finite 2-groups such that $\gamma_{2}(G)$ is elementary abelian of order 16. Also let $\mathrm{Z}(G) \leq \gamma_{2}(G)$. As in the case of odd primes, we have $b(G) \geq 3$ in this case too. If $b(G)=4$, then $\mathrm{K}(G)=\gamma_{2}(G)$. So we assume that $b(G)=3$. Then it follows from Theorem 7.2 that the nilpotency class of $G$ is either 2 or 3 , and $|G| \geq 2^{7}$. If the nilpotency class of $G$ is 2, then the assertion follows from Lemmas 7.4-7.8. If the nilpotency class of $G$ is 3 , then the assertion holds from Lemma [7.3, and the proof is complete.

\section{ExAmples}

In this section we present various types of examples of groups which occur in our study above for $p \geq 3$. Examples of 2-groups are evident from GAP computations.

Groups of class 2. Let $F$ be the freest $p$-group of nilpotency class 2 and exponent $p$ on 4 generators, $a, b, c, d$ (say), where $p$ is an odd prime. Let $R:=\langle[b, d],[a, d]\rangle$. Then $G:=F / R$ is a group of nilpotency class 2 and order $p^{8}$ such that $\mathrm{K}(G) \neq \gamma_{2}(G)$. If we take $R_{1}:=$ $\left\langle[a, b][c, d],[a, c][b, d]^{r}\right\rangle$, where $r$ is any fixed non-square integer modulo p. Then it follows from [18. Theorem 1.2] that $G_{1}:=F / R_{1}$ is a group of order $p^{8}$, nilpotency class 2 and conjugate type $\left\{1, p^{3}\right\}$. For $p$-groups $G, p$ odd, of nilpotency class 2 and order at least $p^{9}$, we know that $\mathrm{K}(G)=\gamma_{2}(G)$. Such examples of order $\geq p^{10}$ can be constructed by taking a central product of the group $G:=F / R$ and any finite extraspecial $p$-group $K$ amalgamating $\langle[\bar{c}, \bar{d}]\rangle=\gamma_{2}(K)$. Constructing such examples of order $p^{9}$ is also easy, as explained in the proof of Lemma 3.4 .

Groups of class 3 . We present five types of $p$-group of nilpotency class 3 and order $p^{7}$, where $p$ is an odd prime. Consider the group presented as

$$
\begin{gathered}
G=\left\langle\alpha_{1}, \alpha_{2}, \alpha_{3}, \alpha_{4}, \alpha_{5}, \alpha_{6}, \gamma\right|\left[\alpha_{2}, \alpha_{1}\right]=\alpha_{4},\left[\alpha_{3}, \alpha_{1}\right]=\alpha_{5},\left[\alpha_{3}, \alpha_{2}\right]=\alpha_{6}, \\
{\left[\alpha_{4}, \alpha_{2}\right]=\gamma,\left[\alpha_{5}, \alpha_{3}\right]=\gamma,\left[\alpha_{5}, \alpha_{2}\right]=\gamma,\left[\alpha_{6}, \alpha_{1}\right]=\gamma, \alpha_{1}{ }^{p}=\gamma,} \\
\left.\alpha_{i}{ }^{p}=\gamma^{p}=1(2 \leq i \leq 6)\right\rangle .
\end{gathered}
$$

Notice that $|\mathrm{Z}(G)|=p$ and $\mathrm{K}(G)=\gamma_{2}(G)$.

The following group $G$ is such that $|Z(G)|=p^{2}=\left|\gamma_{3}(G)\right|$ and $\mathrm{K}(G)=\gamma_{2}(G)$.

$$
\begin{gathered}
G=\left\langle\alpha_{1}, \alpha_{2}, \alpha_{3}, \alpha_{4}, \alpha_{5}, \alpha_{6}, \gamma\right|\left[\alpha_{i}, \alpha_{1}\right]=\alpha_{i+2},\left[\alpha_{5}, \alpha_{1}\right]=\gamma \\
\left.\alpha_{1}^{p}=\alpha_{i}^{p}=\alpha_{5}^{p}=\alpha_{6}^{p}=\gamma^{p}=1(2 \leq i \leq 4)\right\rangle
\end{gathered}
$$


The next group $G$ is such that $|Z(G)|=p^{2},\left|\gamma_{3}(G)\right|=p$ and $\mathrm{K}(G)=\gamma_{2}(G)$.

$$
\begin{gathered}
G=\left\langle\alpha_{1}, \alpha_{2}, \alpha_{3}, \alpha_{4}, \alpha_{5}, \alpha_{6}, \gamma\right|\left[\alpha_{2}, \alpha_{1}\right]=\alpha_{4},\left[\alpha_{3}, \alpha_{1}\right]=\alpha_{5},\left[\alpha_{3}, \alpha_{2}\right]=\alpha_{6}, \\
\left.\left[\alpha_{4}, \alpha_{2}\right]=\gamma,\left[\alpha_{5}, \alpha_{3}\right]=\gamma, \alpha_{1}{ }^{p}=\gamma, \alpha_{i}{ }^{p}=\gamma^{p}=1(2 \leq i \leq 6)\right\rangle .
\end{gathered}
$$

We now present a group $G$ such that $|Z(G)|=p^{3},\left|\gamma_{3}(G)\right|=p$ and $\mathrm{K}(G) \neq \gamma_{2}(G)$.

$$
\begin{array}{r}
G=\left\langle\alpha_{1}, \alpha_{2}, \alpha_{3}, \alpha_{4}, \alpha_{5}, \alpha_{6}, \gamma\right|\left[\alpha_{2}, \alpha_{1}\right]=\alpha_{4},\left[\alpha_{3}, \alpha_{1}\right]=\alpha_{5}, \\
\left.\left[\alpha_{3}, \alpha_{2}\right]=\alpha_{6},\left[\alpha_{4}, \alpha_{1}\right]=\gamma, \alpha_{i}^{p}=\gamma^{p}=1(1 \leq i \leq 6)\right\rangle .
\end{array}
$$

Finally we present a group $G$ such that $|Z(G)|=p^{3},\left|\gamma_{3}(G)\right|=p^{2}$ and $\mathrm{K}(G) \neq \gamma_{2}(G)$.

$$
\begin{array}{r}
G=\left\langle\alpha_{1}, \alpha_{2}, \alpha_{3}, \alpha_{4}, \alpha_{5}, \alpha_{6}, \gamma\right|\left[\alpha_{2}, \alpha_{1}\right]=\alpha_{4},\left[\alpha_{3}, \alpha_{1}\right]=\alpha_{5}, \\
\left.\left[\alpha_{4}, \alpha_{1}\right]=\alpha_{6},\left[\alpha_{4}, \alpha_{2}\right]=\gamma, \alpha_{i}^{p}=\gamma^{p}=1(1 \leq i \leq 6)\right\rangle .
\end{array}
$$

Groups of class 4 . We present two types of $p$-groups of nilpotency class 4 and order $p^{7}$, where $p$ is an odd prime. Consider the group presented as

$$
\begin{aligned}
G=\left\langle\alpha_{1}, \alpha_{2}, \alpha_{3}, \alpha_{4}, \alpha_{5}, \alpha_{6}, \gamma\right|\left[\alpha_{2}, \alpha_{1}\right]=\alpha_{4},\left[\alpha_{4}, \alpha_{1}\right]=\alpha_{5},\left[\alpha_{4}, \alpha_{2}\right]=\alpha_{6}, \\
\\
{\left.\left[\alpha_{4}, \alpha_{3}\right]=\left[\alpha_{5}, \alpha_{1}\right]=\left[\alpha_{6}, \alpha_{2}\right]=\gamma,\left[\alpha_{3}, \alpha_{1}\right]=\gamma, \alpha_{i}^{p}=\gamma^{p}=1(2 \leq i \leq 6)\right\rangle . }
\end{aligned}
$$

For this group $|Z(G)|=p$ and $\mathrm{K}(G)=\gamma_{2}(G)$.

The following is a group $G$ such that $|Z(G)|=p^{2}$ and $\mathrm{K}(G)=\gamma_{2}(G)$.

$$
\begin{gathered}
G=\left\langle\alpha_{1}, \alpha_{2}, \alpha_{3}, \alpha_{4}, \alpha_{5}, \alpha_{6}, \gamma\right|\left[\alpha_{2}, \alpha_{1}\right]=\alpha_{4},\left[\alpha_{4}, \alpha_{1}\right]=\alpha_{5},\left[\alpha_{4}, \alpha_{2}\right]=\alpha_{6}, \\
\left.\left[\alpha_{3}, \alpha_{2}\right]=\left[\alpha_{5}, \alpha_{1}\right]=\gamma, \alpha_{1}{ }^{p}=\gamma, \alpha_{i}{ }^{p}=\gamma^{p}=1(2 \leq i \leq 6)\right\rangle .
\end{gathered}
$$

We conclude with the remark that there are total 159 groups $G$ of order $5^{7}$ such that $\gamma_{2}(G)$ is elementary abelian of order $5^{5}$, and $\mathrm{K}(G) \neq \gamma_{2}(G)$ for 141 such groups.

\section{REFERENCES}

[1] W. Bosma, J. Cannon and C. Playoust, The Magma algebra system. I. The user language, J. Symbolic Comput., 24 (1997), 235-265.

[2] R. R. Dark and M. L. Newell, On conditions for commutators to form a subgroup, J. London Math. Soc. (2) $\mathbf{1 7}$ (1978), 251-262.

[3] The GAP Group, Groups Algorithms and Programming, version 4.8 .10 (2018), available at http://www.gap-system.org

[4] D. Gorenstein, Finite Groups, AMS Chelsea Pub., Second Ed. (1980).

[5] Gustavo A. Fernandez-Alcober and I. de las Heras, Commutators in finite p-groups with 2-generator derived subgroup, Israel J. Math. 232 (2019), 109-124.

[6] N. Gavioli, A. Mann, V. Monti, A. Previtali and C. M. Scoppola. Groups of prime power order with many conjugacy classes. J. Algebra 202 (1998), 129-141.

[7] R. M. Guralnick, Commutators and commutator subgroups, Adv. Math. 45 (1982), 319-330.

[8] R. M. Guralnick, Generation of the lower central series, Glasgow Math. J. 23 (1982), 15-20.

[9] R. M. Guralnick, Generation of the lower central series II, Glasgow Math. J. 25 (1984), 193-201.

[10] P. Hall, The classification of prime power groups, Journal für die reine und angewandte Mathematik 182 (1940), 130-141.

[11] P. Hall, Finite-by-nilpotent groups, Proc. Cambridge Phil. Soc. 52 (1956), 611-616.

[12] I. de las Heras, Commutators in finite p-groups with 3-generator derived subgroup, J. Algebra 546 (2020), 201-217.

[13] Iker de las Heras and M. Morigi, Lower central words in finite p-groups, arXiv:1907.11479

[14] R. James, The groups of order $p^{6}$ ( $p$ an odd prime), Math. Comp 34 (1980), 613-637.

[15] L.-C. Kappe and R. F. Morse, On commutators in p-groups, J. Group Theory 8 (2005), 415-429. 
[16] L.-C. Kappe and R. F. Morse, On commutators in groups, in Groups St. Andrews 2005. Vol. 2, London Mathematical Society Lecture Note Series, Vol. 340, Cambridge University Press, Cambridge, 2007, pp. 531-558.

[17] I. D. Macdonald, The Theory of Groups, Clarendon Press, Oxford, (1968).

[18] T. K. Naik and M. K. Yadav, Finite p-groups of conjugate type $\left\{1, p^{3}\right\}$. J. Group Theory 21 (2018), 65-82.

[19] G. Parmeggiani and B. Stellmacher, p-Groups of small breadth, J. Algebra 213 (1999), 52-68.

[20] D. M. Rodney, On cyclic derived subgroups, J. London Math. Soc. 8 (1974), 642-646.

[21] D. M. Rodney, Commutators and abelian groups, J. Aust. Math. Soc. 24 (1977), 79-91.

[22] B. Wilkens, 2-groups of breadth 3, J. Algebra 318 (2007), 202-224.

School of Mathematics, Harish-Chandra Research Institute, HBni, Chhatnag Road, Jhunsi, AlLAHABAD - 211 019, INDIA

E-mail address: rahulkaushik@hri.res.in

School of Mathematics, Harish-Chandra Research Institute, HBni, Chhatnag Road, Jhunsi, AlLAHABAD - 211 019, INDIA

E-mail address: myadav@hri.res.in 